\title{
Identifying a Higgsino-like NLSP in the context of a keV-scale gravitino LSP
}

\author{
Juhi Dutta $\odot,{ }^{*}$ Biswarup Mukhopadhyaya, ${ }^{\dagger}$ and Santosh Kumar Rai ${ }^{\ddagger}$ \\ Regional Centre for Accelerator-based Particle Physics, Harish-Chandra Research Institute, HBNI, \\ Chhatnag Road, Jhusi, Allahabad 211019, India
}

(Received 28 January 2020; accepted 19 March 2020; published 22 April 2020)

\begin{abstract}
The presence of a Higgsino-like neutralino next-to-lightest sparticle (NLSP) and a keV scale gravitino $(\tilde{G})$ LSP opens up new decay modes of the NLSP, mainly to a Higgs/ $Z$ boson and the LSP. Besides, a keVscale gravitino as a warm dark matter candidate salvages a relatively light Higgsino-like NLSP from dark matter constraints. We focus on the prospects of observing at least one $\mathrm{b}$ jet and two opposite sign leptons with a large missing transverse momenta $\left(\geq 1 b+\ell^{+} \ell^{-}+\mathbb{E}_{T}\right)$ signal at the LHC. A distinguishing feature of this scenario is the production of longitudinal $Z$ bosons in neutralino decays, unlike in the case of gauginolike neutralinos, where the $Z$ is mostly transverse. The polarization information of the parent $Z$ boson gets reflected in the angular distributions of the decay leptons and in some other variables derived therefrom.
\end{abstract}

DOI: 10.1103/PhysRevD.101.075040

\section{INTRODUCTION}

In the light of the $13 \mathrm{TeV}$ LHC run 2 results [1,2], both experimentalists and theorists are leaving no stone unturned to interpret results of different supersymmetric (SUSY) scenarios, primarily to ensure no glaring gaps are left in the current search strategies such that the signals may slip through them. Although bounds on sparticle masses from LHC are steadily increasing, they are derived in the context of simplified scenarios. However, generic features on collider signals are helpful to investigate, if such features reflect the spectrum and the composition of the superparticle states.

The $R$-parity conserving minimal supersymmetric standard model (MSSM) ensures a stable, neutral, colorless dark matter (DM) candidate, generally the lightest neutralino $\left(\tilde{\chi}_{1}^{0}\right)$, which is the lightest SUSY particle (LSP). Depending on its composition, it could be either a democratic admixture of gaugino and Higgsino states or dominantly binolike, winolike, or Higgsino-like. The DM composition faces stringent constraints from direct search data and indirect evidence as well as from relic density measurements by Planck [3]. For example, a bino-dominated $\tilde{\chi}_{1}^{0}$ with an appropriate small admixture of Higgsinos can by and large

\footnotetext{
*juhidutta@hri.res.in biswarup@hri.res.in

skrai@hri.res.in
}

Published by the American Physical Society under the terms of the Creative Commons Attribution 4.0 International license. Further distribution of this work must maintain attribution to the author(s) and the published article's title, journal citation, and DOI. Funded by SCOAP. be consistent with relic density as well as direct search constraints. However for a winolike LSP, SU (2)-driven annihilation channels lead to strong constraints from $\gamma$-ray [4] as well as positron data [5]. A light Higgsino-like LSP is disfavored from direct dark matter searches [6,7]. Conversely, the relic density being inversely proportional to the annihilation cross section, shows an underabundance for wino or Higgsino-like LSP, whereas a binolike LSP can lead to an overabundance unless coannihilation occurs at an adequate rate. Mixed bino-Higgsino or bino-wino dark matter scenarios could be more consistent from these standpoints [8]. The presence of a lighter particle such as a gravitino or an axino as the lightest SUSY particle relaxes these constraints on the composition of the lightest neutralino $\tilde{\chi}_{1}^{0}$, which now serves as the next-to-lightest sparticle (NLSP). Gravitinos are the LSP in models like gauge mediated supersymmetry breaking with low scale of SUSY breaking. Here, the gravitino mass scale may be lighter than the MSSM sparticle masses if the scale of SUSY breaking is light enough to allow a light gravitino mass governed by [9-11],

$$
m_{\tilde{G}}=\frac{\langle F\rangle}{\sqrt{3} M_{\mathrm{Pl}}},
$$

where $m_{\tilde{G}}$ is the mass of the gravitino, $\langle F\rangle$ is the SUSY breaking scale, and $M_{\mathrm{Pl}}$ is the Planck scale. This means that depending on the SUSY breaking scale $\langle F\rangle$, the gravitino can be as light as $\mathcal{O}(\mathrm{keV})$. A light gravitino with mass of few $\mathrm{keV}$ is motivated to be a warm dark matter candidate [12-14]. In this work, we consider the LHC signals of a Higgsino-dominated NLSP, where one has such a light gravitino LSP. 
Signals for a dominantly Higgsino-like $\tilde{\chi}_{1}^{0}$ NLSP with a gravitino LSP has been studied by experimental collaborations at the LHC. A primarily Higgsino-like $\tilde{\chi}_{1}^{0}$ NLSP is found to decay dominantly to either the Higgs or $Z$ boson along with gravitino. Since the light standard model (SM) like Higgs has the largest decay probability to $b \bar{b}$, this leads to a final state dominated by hard $b$-tagged jets along with a large missing transverse momentum (MET) $\mathbb{E}_{T}$ and additional light jets/leptons arising from an accompanying $Z$ boson. Signatures for the Higgsino-like NLSP's have been studied in the context of Tevatron $[15,16]$ and also at LHC, where both CMS [17] and ATLAS [18] have looked at multiple $b$ jets + MET, dilepton, and multilepton states to constrain a Higgsino-like NLSP scenario.

In this work, we aim to study signatures of a low-lying Higgsino sector in the presence of a light gravitino LSP with emphasis on determining how the NLSP nature can be convincingly identified. To do this, one would like to reconstruct the decay products of the NLSP. As the Higgsino NLSP would decay to a light Higgs or a $Z$ boson, we may be able to observe their properties by appropriately reconstructing the Higgs boson through the $b$ jets arising from its decay as well as the $Z$ boson through the opposite sign dilepton pair from the gauge boson's decay, respectively. We note here a very important and interesting feature of the decay of the NLSP. It is expected that the $Z$ boson arising from the Higgsino-like $\tilde{\chi}_{1}^{0}$ decay would be dominantly longitudinal (Goldstone boson), primarily following the equivalence theorem where, after electroweak symmetry breaking, the neutral Goldstone boson constitutes the longitudinal mode of the $Z$ boson responsible for its mass. This property if observed in the decay of the NLSP would exclusively point towards the presence of a Higgsino-like $\tilde{\chi}_{1}^{0}$, helping us identify the nature of the NLSP. The direct production of the electroweak neutralino NLSP would be limited at the LHC as their mass becomes larger. However, the property of the NLSP could still be studied if they are produced in cascade decays of strongly interacting sparticles. We therefore study the effect of including the strong sector in exploring the compositions of the NLSP as well as from the direct production of the low-lying electroweakinos (still allowed by experiments) and propose some new kinematic observables which help identify the NLSP. Thus, the salient points of our study are as follows:

(i) We consider a naturally compressed low-lying Higgsino sector as well as partially and/or fully compressed spectra with the strongly interacting sparticles sitting above the NLSP. The sparticles decay via cascades to the NLSP, which further decays to a Higgs and a $Z$ boson, thereby giving rise to at least $1 b$ jet and opposite-sign same flavor dileptons along with missing transverse energy in the final state.

(ii) The characteristic features of a longitudinal $Z$ boson arising from decay of the Higgsino-like $\tilde{\chi}_{1}^{0}$ are studied by utilizing angular variables of the negatively charged lepton. In order to distinguish it from transversely polarized $Z$ bosons coming from other sources, we compare our results with the complementary admixture of NLSP, especially gaugino-dominated neutralinos as well as the SM background.

(iii) We observe that for a spectrum with a heavy NLSP, reflecting overall compression with respect to the strong sector leads to an increased fraction of the longitudinal mode in the $Z$ boson arising from the NLSP decay.

(iv) New observables enhancing the asymmetry in the angular distributions of the negatively charged lepton have been proposed in order to characterize a longitudinally polarized $Z$ boson in comparison to a transversely polarized $Z$ boson. Such observables distinctly vary, depending on the Higgsino-gaugino admixture of the NLSP, and crucially capture the effect of the equivalence theorem for a heavy NLSP.

The paper is organized as follows. In Sec. II, we discuss the current scenario with the Higgsino NLSP and gravitino LSP followed by the decay properties of the Higgsinos in Sec. III. In Sec. IV, we discuss the experimental status of a Higgsino-like NLSP with a light gravitino LSP at LHC. In Sec. V, we choose some benchmarks to study the available parameter space. We perform the collider study and discuss our results at the high luminosity run of LHC in Sec. VI. In Sec. VII, we distinguish between the features of longitudinal and transverse gauge bosons. Section VIII summarizes the main conclusions of our work.

\section{HIGGSINO-DOMINATED NLSP WITH KEV LSP}

In our work, we discuss light Higgsino-like NLSP as a possible consequence of a general phenomenological MSSM. Since no hint of SUSY has yet shown up at direct searches, various possible configurations of the lightest neutralino, $\tilde{\chi}_{1}^{0}$, leading to distinct signals at colliders are of interest. Such a light Higgsino-like $\tilde{\chi}_{1}^{0}$ is characterized by a low $(\lesssim 800 \mathrm{GeV}) \mu$ parameter and heavy bino, wino soft mass parameters, i.e., $|\mu| \ll M_{1}, M_{2} . \mu$ in the aforesaid range is also a preferred choice from the angle of naturalness [19-24]. However, $\tilde{\chi}_{1}^{0}$ may not be the LSP in many situations. In such cases, there can be several other candidates for LSP such as gravitinos, axinos, sneutrinos, etc.

The gravitino is the spin $\frac{3}{2}$ superpartner of the spin 2 graviton in local SUSY. Upon spontaneous SUSY breaking, there arises a massless Weyl fermion known as the goldstino $(\tilde{G})$, owing to the breaking of the fermionic generators of SUSY. After electroweak symmetry breaking, the gravitino acquires mass by absorbing the goldstino, which form the spin $\frac{1}{2}$ components of the massive gravitino. We henceforth approximate a light gravitino by the goldstino using the equivalence theorem. 
The goldstino $(\tilde{G})$ Lagrangian is [9],

$$
\mathcal{L}_{\text {goldstino }}=i \tilde{G}^{\dagger} \bar{\sigma}^{\mu} \partial_{\mu} \tilde{G}-\frac{1}{\langle F\rangle} \tilde{G} \partial_{\mu} j^{\mu}+\text { c.c. },
$$

where $j^{\mu}$ refers to the supercurrent involving all other sparticles and SM particles. The couplings of the gravitino to fermion-sfermion, gauge boson-gauginos are computed in Ref. [25]. Having said this, we briefly discuss the couplings and decays of a Higgsino-like NLSP in the presence of a light $\tilde{G}$ before moving on to our numerical analysis.

\section{HIGGSINO NLSP DECAYS}

A Higgsino-like $\tilde{\chi}_{1}^{0}$ is characterized by a large Higgsino fraction with suppressed wino and bino fractions, i.e., $\mu<M_{1}, M_{2}$. In the presence of a light $\tilde{G}$ LSP, the Higgsino-like $\tilde{\chi}_{1}^{0}$ NLSP decays to either a Higgs $(h)$ or a $Z$ boson and $\tilde{G}$. Absence of a large bino component leads to a rather suppressed photon mode unless there is substantial gaugino-bino-Higgsino admixture [16]. However, the photon mode may dominate in case of very light Higgsinos, where the decay to the Higgs or $Z$ boson is phase space suppressed. As the coupling of a gravitino to other particles are inversely proportional to its $\operatorname{mass}\left(m_{\tilde{G}}\right)$, a lighter gravitino has stronger couplings as compared to a heavier one. For any sparticle $\tilde{X}$ decaying into its SM partner $X$ and the gravitino, the width is given by [9]

$$
\Gamma(\tilde{X} \rightarrow X \tilde{G})=\frac{m_{\tilde{X}}^{5}}{48 \pi M_{P}^{2} m_{\tilde{G}}^{2}}\left(1-\frac{m_{X}^{2}}{m_{\tilde{X}}^{2}}\right)^{4},
$$

where $m_{X(\tilde{X})}$ refers to the mass of $X(\tilde{X})$. As we are interested in the decay of the neutralino NLSP to the gravitino, the composition of the lightest neutralino becomes an essential characteristic as it would determine what the NLSP finally decays to. The neutralino mass matrix in the basis $\left(\tilde{B}, \tilde{W}_{3}, \tilde{H}_{d}^{0}, \tilde{H}_{u}^{0}\right)$ is as follows [9]:

$$
M^{n}=\left(\begin{array}{cccc}
M_{1} & 0 & -M_{Z} s_{W} c_{\beta} & M_{Z} s_{W} s_{\beta} \\
0 & M_{2} & M_{Z} c_{W} c_{\beta} & -M_{Z} c_{W} s_{\beta} \\
-M_{Z} s_{W} c_{\beta} & M_{Z} c_{W} c_{\beta} & 0 & -\mu \\
M_{Z} s_{W} s_{\beta} & -M_{Z} c_{W} s_{\beta} & -\mu & 0
\end{array}\right) .
$$

Here, $s_{W}=\sin \theta_{W}, c_{W}=\cos \theta_{W}$, where $\theta_{W}$ is the weak mixing angle, whereas $s_{\beta}=\sin \beta, c_{\beta}=\cos \beta$, where $\tan \beta=\frac{v_{u}}{v_{d}}$ refers to the ratio of the vacuum expectation value's of the up-type Higgs doublet $\left(H_{u}\right)$ and the downtype Higgs doublet $H_{d}$. Diagonalizing the symmetric mass matrix $M^{n}$ using a unitary matrix $N$ lead to the neutralino mass eigenstates $\tilde{\chi}_{i}^{0}(i=1, \ldots, 4)$,

$$
N M^{n} N^{T}=\operatorname{diag}\left(m_{\tilde{\chi}_{1}^{0}}, m_{\tilde{\chi}_{2}^{0}}, m_{\tilde{\chi}_{3}^{0}}, m_{\tilde{\chi}_{4}^{0}}\right),
$$

where $m_{\tilde{\chi}_{1}^{0}}<m_{\tilde{\chi}_{2}^{0}}<m_{\tilde{\chi}_{3}^{0}}<m_{\tilde{\chi}_{4}^{0}}$.

The chargino mass matrix $M^{c}$ in the basis $\left(\tilde{W}^{+}, \tilde{H}_{u}^{+}\right)$is as follows [9]:

$$
M^{c}=\left(\begin{array}{cc}
M_{2} & \sqrt{2} M_{W} s_{\beta} \\
\sqrt{2} M_{W} c_{\beta} & \mu
\end{array}\right) .
$$

Since, $M^{c}$ is not a symmetric matrix, we need two unitary matrices $U$ and $V$ to diagonalize the matrix. Hence,

$$
U^{*} M^{c} V^{-1}=\operatorname{diag}\left(m_{\tilde{\chi}_{1}^{ \pm}}, m_{\tilde{\chi}_{2}^{ \pm}}\right),
$$

where $m_{\tilde{\chi}_{1}^{ \pm}}<m_{\tilde{\chi}_{2}^{ \pm}}$. In the limit where $\mu \ll M_{1}, M_{2}$, there are two nearly degenerate Higgsino-like neutralinos along with a degenerate Higgsino-like chargino, leading to a naturally compressed spectrum. The mass eigenvalues at the tree level are $[26,27]$

$$
\begin{aligned}
& m_{\tilde{\chi}_{1}^{ \pm}}=|\mu|\left(1-\frac{M_{W}^{2} \sin 2 \beta}{\mu M_{2}}\right)+\mathcal{O}\left(M_{2}^{-2}\right) \\
& m_{\tilde{\chi}_{1,2}^{0}}= \pm \mu-\frac{M_{Z}^{2}}{2}(1 \pm \sin 2 \beta)\left(\frac{\sin ^{2} \theta_{W}}{M_{1}}+\frac{\cos ^{2} \theta_{W}}{M_{2}}\right) .
\end{aligned}
$$

In the presence of a light $\tilde{G}$, the following additional decay modes open up for the Higgsino-like chargino and neutralinos:

$$
\begin{aligned}
\tilde{\chi}_{1}^{ \pm} & \rightarrow W^{ \pm} \tilde{G} \\
\tilde{\chi}_{2}^{0} & \rightarrow h \tilde{G}, Z \tilde{G} \\
\tilde{\chi}_{1}^{0} & \rightarrow h \tilde{G}, Z \tilde{G},
\end{aligned}
$$

where the $Z$ boson from a neutralino decay is mostly longitudinal. The squared couplings of the Higgsino-like neutralinos and chargino, to the gravitino $(\tilde{G})$ LSP are [28]

$$
\begin{aligned}
\left|g_{\tilde{G} \tilde{\chi}_{i}^{0} H_{k}}\right|^{2} & =\left|e_{k} N_{i 3}+d_{k} N_{i 4}\right|^{2}\left(M_{\mathrm{Pl}} m_{\tilde{G}}\right)^{-2}, \\
\left|g_{\tilde{G} \tilde{\chi}_{1}^{ \pm} H^{ \pm}}\right|^{2} & =\left(\left|V_{12}^{2}\right| \cos ^{2} \beta+\left|U_{12}^{2}\right| \sin ^{2} \beta\right)\left(M_{\mathrm{Pl}} m_{\tilde{G}}\right)^{-2},
\end{aligned}
$$

TABLE I. Relevant range of the input parameters for the parameter-space scan to study the decay probabilities of the lightest neutralino is shown. We keep other parameters at fixed values which include: $M_{1}=2 \mathrm{TeV}, M_{2}=2 \mathrm{TeV}, M_{3}=1.917 \mathrm{TeV}, M_{Q_{3}}=$ $2.8 \mathrm{TeV}, M_{U_{3}}=2.8 \mathrm{TeV}, M_{A}=2.5 \mathrm{TeV}, A_{t}=3 \mathrm{TeV}$, and $m_{\tilde{G}}=1 \mathrm{keV}$.

\begin{tabular}{lccc}
\hline \hline Parameters & $|\mu|(\mathrm{TeV})$ & $\operatorname{sign}(\mu)$ & $\tan \beta$ \\
\hline Values & $0.2-1.5$ & \pm 1 & $2-45$ \\
\hline \hline
\end{tabular}



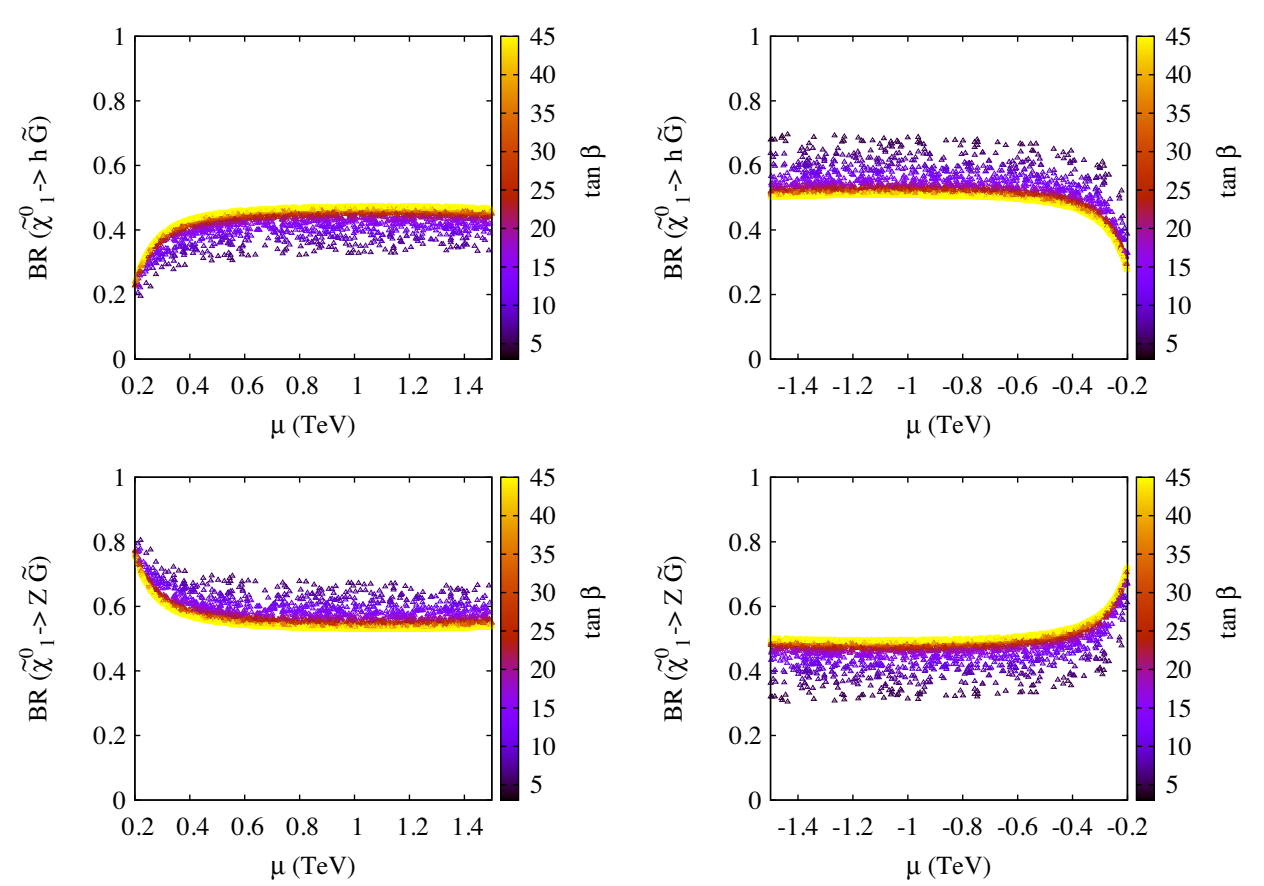

FIG. 1. Variation of $\tilde{\chi}_{1}^{0}$ decay into a Higgs (top panel) or $Z$ boson (bottom panel) along with the $\tilde{G}$ LSP with $\mu$ and tan $\beta$ in the colored palette. The parameters of the scan are listed in Table I.

where $i=1,2$ and $k=1,2,3$, such that $H_{k}=h, H, A$, respectively. The coefficients $e_{k}$ and $d_{k}$ are as below,

$$
\begin{array}{llr}
e_{1}=\cos \alpha, & e_{2}=-\sin \alpha, & e_{3}=-\sin \beta \\
d_{1}=-\sin \alpha, & d_{2}=-\cos \alpha, & d_{3}=\cos \beta,
\end{array}
$$

and $N_{i j}$ refer to the $(i j)^{\text {th }}$ entry of the neutralino mixing matrix $N, \alpha$ is the mixing angle between the $C P$-even Higgs bosons, $h$ and $H$. In the decoupling limit, i.e., $m_{A} \gg m_{h}$, $\beta-\alpha \sim \pi / 2$ (where, $0<\beta<\pi$ and $-\pi<\alpha<0$ ), the lightest $C P$-even Higgs boson $(h)$ behaves like the SM Higgs boson [9]. The partial decay widths of the lightest neutralino $\tilde{\chi}_{1}^{0}$ are as follows $[15,29]$ :

$$
\begin{gathered}
\Gamma\left(\tilde{\chi}_{1}^{0} \rightarrow h \tilde{G}\right) \propto\left|N_{14} \sin \alpha-N_{13} \cos \alpha\right|^{2}\left(M_{\mathrm{P} 1} m_{\tilde{G}}\right)^{-2} \\
\Gamma\left(\tilde{\chi}_{1}^{0} \rightarrow Z \tilde{G}\right) \propto\left(\left|N_{11} \sin \theta_{W}-N_{12} \cos \theta_{W}\right|^{2}\right. \\
\left.\quad+\frac{1}{2}\left|N_{14} \cos \beta-N_{13} \sin \beta\right|^{2}\right)\left(M_{\mathrm{Pl}} m_{\tilde{G}}\right)^{-2}
\end{gathered}
$$

The terms proportional to $N_{14}$ and $N_{13}$ denote the Goldstone couplings. ${ }^{1}$ In the decoupling limit, $\sin \alpha=$ $-\cos \beta$ and $\cos \alpha=\sin \beta$; thus, Eq. (11) reduces to

\footnotetext{
${ }^{1}$ See the discussion at the beginning of Sec. VII for the decay of the $Z$ boson and the polarization of the gauge bosons.
}

$\Gamma\left(\tilde{\chi}_{1}^{0} \rightarrow h \tilde{G}\right) \propto\left|N_{14} \cos \beta+N_{13} \sin \beta\right|^{2}\left(M_{\mathrm{Pl}} m_{\tilde{G}}\right)^{-2}$.

The interference term between the gaugino and the Higgsino vanishes [30] in the above decay mode due to the polarization states being physical states. We note that for a Higgsino-like $\tilde{\chi}_{1}^{0}, N_{13} \simeq-N_{14}$ for $\mu>0$, whereas for $\mu<0, N_{13} \sim N_{14}$ [15]. This leads to an increase in $\Gamma\left(\tilde{\chi}_{1}^{0} \rightarrow h \tilde{G}\right)$ as evident from Eq. (13).

\section{A. Branching ratios}

The decay branching ratios of the Higgsino-dominated $\tilde{\chi}_{1}^{0}$ NLSP are governed mainly by values of $\mu$ and $\tan \beta$. Table I summarizes the relevant parameter ranges for the scan performed using the package SPheno-v3.3.6 [31,32]. In Fig. 1, we show the regions of the parameter space, having the different branching ratios of the $\tilde{\chi}_{1}^{0}$. We discuss the salient points of the parameter space as below:

(i) The branching fractions to the Higgs and $Z$ mode decreases with an increase in the gaugino admixture in the NLSP at higher values of $\mu$ and $\tan \beta$ (as $\mu$ gets closer to the choice of $M_{1}$ and $M_{2}$ shown in Table I). This defines a range of the parameter space with comparable branching ratios for the Higgs and $Z$ boson decay modes of the $\tilde{\chi}_{1}^{0}$ NLSP.

(ii) For $|\mu|>400 \mathrm{GeV}$,

$$
\operatorname{BR}\left(\tilde{\chi}_{1}^{0} \rightarrow h \tilde{G}\right) \simeq \operatorname{BR}\left(\tilde{\chi}_{1}^{0} \rightarrow Z \tilde{G}\right),
$$

for large values of $\tan \beta(\tan \beta \geq 25)$ with small differences depending on $\operatorname{sign}(\mu)$. For $\operatorname{sign}(\mu)=+1$, 
$\mathrm{BR}\left(\tilde{\chi}_{1}^{0} \rightarrow h \tilde{G}\right) \simeq 0.47, \quad \operatorname{BR}\left(\tilde{\chi}_{1}^{0} \rightarrow Z \tilde{G}\right) \simeq 0.53$,

whereas for $\operatorname{sign}(\mu)=-1$,

$$
\operatorname{BR}\left(\tilde{\chi}_{1}^{0} \rightarrow h \tilde{G}\right) \simeq 0.54, \quad \operatorname{BR}\left(\tilde{\chi}_{1}^{0} \rightarrow Z \tilde{G}\right) \simeq 0.46 .
$$

(iii) For $\mu<400 \mathrm{GeV}$ and $\tan \beta \simeq 5$,

$$
\operatorname{BR}\left(\tilde{\chi}_{1}^{0} \rightarrow h \tilde{G}\right) \simeq 0.30, \quad \operatorname{BR}\left(\tilde{\chi}_{1}^{0} \rightarrow Z \tilde{G}\right) \simeq 0.70 .
$$

due to $N_{13}=-N_{14}$, such that the $Z$ mode dominates over the Higgs mode. Whereas for $-\mu>400 \mathrm{GeV}$ and $\tan \beta \simeq 5$,

$$
\operatorname{BR}\left(\tilde{\chi}_{1}^{0} \rightarrow h \tilde{G}\right) \simeq 0.67, \quad \operatorname{BR}\left(\tilde{\chi}_{1}^{0} \rightarrow Z \tilde{G}\right) \simeq 0.33,
$$

where $N_{13}=N_{14}$ and the Higgs and $Z$ decay modes of the $\tilde{\chi}_{1}^{0}$ NLSP dominate over the others, respectively.

In addition, as the $\tilde{\chi}_{1}^{0}$ becomes more gauginolike, the additional decay mode of $\gamma \tilde{G}$ would also open up and subsequently dominate the branching probabilities [16].

\section{EXISTING LHC LIMITS}

The current bounds on the light Higgsinos as NLSP and $\tilde{G}$ LSP are well studied at LHC for a light gravitino $\left(m_{\tilde{G}}=1 \mathrm{GeV}\right.$ [33]) assuming prompt decays. ${ }^{2}$ The relevant analyses are summarized in Table II, and we list the constraints from LHC on the Higgsinos as well as on the strong sector sparticles as relevant for our study below:

(i) Higgsinos: ATLAS and CMS impose stringent limits on the mass of the Higgsinos from searches involving multiple b jets/leptons along with large $\mathbb{E}_{T}$ assuming specific branching probabilities for its decay. The following are the exclusion limits on the Higgsino masses [17,33]:

$$
\begin{aligned}
\operatorname{BR}\left(\tilde{\chi}_{1}^{0} \rightarrow h \tilde{G}\right) \sim 1.0: m_{\tilde{\chi}_{1}^{0}} & \leq 880 \mathrm{GeV}(\text { ATLAS }) ; \\
m_{\tilde{\chi}_{1}^{0}} & \leq 760 \mathrm{GeV}(\mathrm{CMS}) . \\
\operatorname{BR}\left(\tilde{\chi}_{1}^{0} \rightarrow Z \tilde{G}\right) \sim 1.0: m_{\tilde{\chi}_{1}^{0}} & \leq 340 \mathrm{GeV} \text { (ATLAS). }
\end{aligned}
$$

Combined exclusion limits on the Higgsino mass from multiple searches at CMS are as follows [17]:

$$
\begin{aligned}
& \operatorname{BR}\left(\tilde{\chi}_{1}^{0} \rightarrow h \tilde{G}\right) \sim 1.0: m_{\tilde{\chi}_{1}^{0}} \leq 775 \mathrm{GeV}(\mathrm{CMS}) \\
& \operatorname{BR}\left(\tilde{\chi}_{1}^{0} \rightarrow Z \tilde{G}\right) \sim 1.0: m_{\tilde{\chi}_{1}^{0}} \leq 650 \mathrm{GeV}(\mathrm{CMS}) .
\end{aligned}
$$

\footnotetext{
${ }^{2}$ Note that $m_{\tilde{G}}=1 \mathrm{GeV}$ would correspond to a long-lived NLSP as Eq. (3) suggests. As long as the mass of the gravitino would not affect the kinematics of the process, it does not affect the analysis reported in [33].
}

TABLE II. List of experimental searches from LHC reinterpreted for Higgsinos relevant for our current study with $\tilde{G}$ LSP. The rate in each final state is the sum of rates in all channels listed in the corresponding rows.

\begin{tabular}{lccc}
\hline \hline Final state & Contributing channels & ATLAS & CMS \\
\hline $2 / 3 / 4 b+\mathbb{E}_{T}$ & $\tilde{\chi}_{1}^{0} \tilde{\chi}_{1}^{ \pm}, \tilde{\chi}_{2}^{0} \tilde{\chi}_{1}^{ \pm}, \tilde{\chi}_{1}^{+} \tilde{\chi}_{1}^{-}, \tilde{\chi}_{1}^{0} \tilde{\chi}_{2}^{0}$ & {$[33]$} & {$[17]$} \\
$\ell^{+} \ell^{-}+\mathbb{E}_{T}$ & $\tilde{\chi}_{1}^{0} \tilde{\chi}_{1}^{ \pm}, \tilde{\chi}_{2}^{0} \tilde{\chi}_{1}^{ \pm}, \tilde{\chi}_{1}^{+} \tilde{\chi}_{1}^{-}, \tilde{\chi}_{1}^{0} \tilde{\chi}_{2}^{0}$ & & {$[17]$} \\
$\geq 3 \ell+\mathbb{E}_{T}$ & $\tilde{\chi}_{1}^{0} \tilde{\chi}_{1}^{ \pm}, \tilde{\chi}_{2}^{2} \tilde{\chi}_{1}^{ \pm}, \tilde{\chi}_{1}^{+} \tilde{\chi}_{1}^{-}, \tilde{\chi}_{1}^{0} \tilde{\chi}_{2}^{0}$ & & {$[17]$} \\
$h h+\mathbb{E}_{T}$ & $\tilde{g} \tilde{g}$ & & {$[34]$} \\
$4 \ell+\mathbb{E}_{T}$ & $\tilde{\chi}_{1}^{+} \tilde{\chi}_{1}^{-}, \tilde{\chi}_{1}^{ \pm} \tilde{\chi}_{2}^{0}$ & {$[35]$} & \\
$\geq 2 j+\mathbb{E}_{T}$ & $\tilde{g} \tilde{g}, \tilde{q} \tilde{q}$ & {$[36]$} & \\
$b \bar{b}+\mathbb{E}_{T}$ & $\tilde{\chi}_{2}^{0} \tilde{\chi}_{1}^{ \pm}$ & {$[37]$} & \\
$1 \ell+b \bar{b}+\mathbb{E}_{T}$ & $\tilde{\chi}_{1}^{0} \tilde{\chi}_{1}^{ \pm}$ & {$[37]$} & \\
$3 \ell+\mathbb{E}_{T}$ & $\tilde{\chi}_{2}^{0} \tilde{\chi}_{1}^{ \pm}$ & {$[37]$} & \\
$\ell^{ \pm} \ell^{ \pm}+\mathbb{E}_{T}$ & $\tilde{\chi}_{2}^{0} \tilde{\chi}_{1}^{ \pm}$ & {$[37]$} & \\
\hline \hline
\end{tabular}

(ii) Strong sector: Direct limits for a massless gravitino LSP scenario are placed on strong sector sparticles with $\tilde{G}$ LSP from opposite sign dilepton + missing energy searches in ATLAS [38], excluding $m_{\tilde{g}} \leq 1.8 \mathrm{TeV}$ for $m_{\tilde{\chi}_{1}^{0}}<600 \mathrm{GeV}$. Stringent limits also arise from boosted Higgs searches [39] interpreted in terms of a simplified scenario with a light $\tilde{\chi}_{1}^{0} \mathrm{LSP}$, excluding $m_{\tilde{g}} \leq 2.2 \mathrm{TeV}$ for $m_{\tilde{\chi}_{1}^{0}}=1 \mathrm{GeV}$. Other indirect searches, which constrain the above mentioned scenario, are multijets and/or multileptons $+\mathbb{E}_{T}$ searches $[35,36]$, owing to the presence of $h / Z$ from the NLSP decay which give rise to leptons or jets in the final state.

\section{BENCHMARKS FOR OUR ANALYSIS}

We choose representative benchmark points of the allowed parameter space to probe a low-lying Higgsinolike $\tilde{\chi}_{1}^{0}$ NLSP with light $\tilde{G}$ LSP, focusing primarily on promptly decaying $\tilde{\chi}_{1}^{0}$ signals. Our choice of benchmarks is motivated by the underlying aim of uncovering the characteristics of a Higgsino-like NLSP in the presence of a light $\tilde{G}$ LSP. Decays of the strong sector sparticles occur via the following decay modes for a $\mathrm{keV} \tilde{G}$ : for gluinos $\left(m_{\tilde{g}}<m_{\tilde{q}} \leq m_{\tilde{l}}, m_{\tilde{\chi}_{3}^{0}}, m_{\tilde{\chi}_{4}^{0}}, m_{\tilde{\chi}_{2}^{ \pm}}\right)$, the possible decay modes to the NLSP,

$$
\tilde{g} \rightarrow t \bar{t} \tilde{\chi}_{1}^{0}, \quad b \bar{b} \tilde{\chi}_{1}^{0}, \quad t \bar{b} \tilde{\chi}_{1}^{-}, \quad q \bar{q}^{\prime} \tilde{\chi}_{1}^{ \pm}, \quad q \bar{q} \tilde{\chi}_{1}^{0} .
$$

Among these decay modes, owing to the Higgsino-like nature of the NLSP, the interaction strengths are governed by the Yukawa couplings. Hence, the third generation squark channels dominate. For squarks $\left(m_{\tilde{q}}<m_{\tilde{g}} \leq m_{\tilde{l}}\right.$, $\left.m_{\tilde{\chi}_{3}^{0}}, m_{\tilde{\chi}_{4}^{0}}, m_{\tilde{\chi}_{2}^{ \pm}}\right)$, the possible decay modes are

\footnotetext{
${ }^{3}$ The third generation squarks decay would have additional decay modes, such as $\tilde{t}_{1} \rightarrow \tilde{b} W^{+}$and $\tilde{b}_{1} \rightarrow \tilde{t} W^{-}$.
} 
TABLE III. List of benchmarks chosen for our study. Mass parameters are in $\mathrm{GeV}$ unless specified otherwise.

\begin{tabular}{|c|c|c|c|c|c|}
\hline Parameters & BP1 & BP2 & BP3 & BP4 & BP5 \\
\hline$M_{1}$ & 2400 & 800 & 7000 & 2300 & 2400 \\
\hline$M_{2}$ & 2400 & 800 & 7000 & 2300 & 2400 \\
\hline$\mu$ & 800 & 2400 & 700 & 2250 & -800 \\
\hline $\tan \beta$ & 25 & 25 & 25 & 25 & 3.8 \\
\hline$A_{t}$ & 3200 & 3200 & 100 & 3200 & 3740 \\
\hline$m_{A}$ & 2500 & 2500 & 2500 & 2500 & 3000 \\
\hline$m_{h}$ & 125.3 & 125.3 & 127.1 & 124.5 & 122.2 \\
\hline$m_{\tilde{g}}$ & 2806.4 & 2807.1 & 7271.2 & 2840.1 & 2663.3 \\
\hline$m_{\tilde{q}_{L}}$ & 2303.3 & 2300.2 & 7156.4 & 2313.3 & 2280.6 \\
\hline$m_{\tilde{q}_{R}}$ & 2302.2 & 2302.5 & 7155.4 & 2312.5 & 2283.7 \\
\hline$m_{\tilde{t}_{1}}$ & 2357.5 & 2184.8 & 7057.0 & 2509.1 & 1581.1 \\
\hline$m_{\tilde{t}_{2}}$ & 2340.9 & 2370.8 & 7104.0 & 2666.0 & 2271.4 \\
\hline$m_{\tilde{b}_{1}}$ & 2260.9 & 2266.4 & 7102.2 & 2583.4 & 2237.5 \\
\hline$m_{\tilde{b}_{2}}$ & 2299.0 & 2323.9 & 7129.0 & 2630.3 & 2295.6 \\
\hline$m_{\tilde{l}_{L}}$ & 3331.8 & 3326.8 & 7337.2 & 3332.6 & 3329.4 \\
\hline$m_{\tilde{l}_{R}}$ & 3335.6 & 3333.7 & 7336.3 & 3336.3 & 3334.1 \\
\hline$m_{\tilde{\chi}_{1}^{0}}$ & 810.9 & 797.9 & 718.8 & 2211.0 & 1214.8 \\
\hline$m_{\tilde{\chi}_{2}^{0}}$ & -814.4 & 837.8 & -723.7 & -2254.8 & -1217.2 \\
\hline$m_{\tilde{\chi}_{1}^{ \pm}}$ & 812.5 & 837.9 & 720.9 & 2223.1 & 1216.4 \\
\hline$m_{\tilde{\chi}_{2}^{ \pm}}$ & 2415.7 & 2397.3 & 1925.9 & 2350.5 & 2420.9 \\
\hline$m_{\tilde{\chi}_{3}^{0}}$ & 2386.3 & -2394.8 & 1923.6 & 2290.1 & 2392.2 \\
\hline$m_{\tilde{\chi}_{4}^{0}}$ & 2415.6 & 2397.4 & 1925.8 & 2350.5 & 2420.9 \\
\hline$m_{\tilde{G}}(\mathrm{keV})$ & 1.0 & 1.0 & 1.0 & 1.0 & 1.0 \\
\hline $\operatorname{BR}\left(\tilde{\chi}_{1}^{0} \rightarrow h \tilde{G}\right)$ & 0.45 & 0.0 & 0.44 & 0.23 & 0.27 \\
\hline $\operatorname{BR}\left(\tilde{\chi}_{1}^{0} \rightarrow Z \tilde{G}\right)$ & 0.55 & 0.25 & 0.56 & 0.75 & 0.73 \\
\hline $\operatorname{BR}\left(\tilde{\chi}_{1}^{0} \rightarrow \gamma \tilde{G}\right)$ & 0 & 0.75 & 0 & 0.02 & 0 \\
\hline $\operatorname{BR}\left(\tilde{\chi}_{1}^{ \pm} \rightarrow W \tilde{G}\right)$ & 0.024 & 0.0 & 0.003 & 0.0001 & 0.15 \\
\hline $\operatorname{BR}\left(\tilde{\chi}_{1}^{ \pm} \rightarrow W^{*} \tilde{\chi}_{1}^{0}\right)$ & 0.976 & 1.0 & 0.997 & 0.9999 & 0.85 \\
\hline
\end{tabular}

$$
\tilde{q} \rightarrow q \tilde{\chi}_{1}^{0}, \quad q \tilde{\chi}_{2}^{0}, \quad q^{\prime} \tilde{\chi}_{1}^{ \pm}
$$

As discussed in Sec. III A, the dominant decay mode of the $\tilde{\chi}_{1}^{0}$ NLSP is to either a Higgs or a $Z$ boson along with the $\tilde{G}$ LSP which contributes to the missing energy. We wish to study the collider prospects of observing the final state $\geq$ $1 b+\ell^{+} \ell^{-}+\mathbb{E}_{T}$ in the context of the upcoming high luminosity run of the LHC and explore kinematic variables reflecting the composition of the NLSP. We discuss below the characteristic features of each of the chosen benchmarks as shown in Table III. We also include a benchmark BP5 similar to BP1 with a larger branching fraction into the Higgs boson and gravitino mode, which would represent the low $\tan \beta$ and negative $\mu$ region of the parameter space.

For simplicity, $M_{1} \simeq M_{2} \sim 2.3-2.4 \mathrm{TeV}$, such that their contribution to the signal region under study (directly or via cascade decays of strong sector sparticles) is negligible. Among the constraints on the parameter space, the light
Higgs mass is within the range $122-128 \mathrm{GeV}[40,41]$. In all cases, both $\tilde{t}_{1}$ and/or $\tilde{t}_{2}$ are heavy or the trilinear coupling $A_{t}$, is too large to fit the lightest $C P$-even Higgs mass [40-42]. Also, $m_{\tilde{\chi}_{1}^{ \pm}}$adheres to the LEP lower limit of 103.5 GeV [43]. We choose the benchmarks after passing them through the public software CheckMATE [44]. Among the searches implemented in CheckMATE, stringent constraints come from multijet searches by ATLAS [36]. The benchmark points are generated using the spectrum generator SPheno-v3.3.6 [31,32].

\section{LHC SIGNALS}

We now discuss in detail the possible LHC signals arising in the current scenario with a Higgsino-like $\tilde{\chi}_{1}^{0}$ NLSP and keV $\tilde{G}$ LSP. A strong sector sparticles pair produced at $\sqrt{s}=13 \mathrm{TeV}$ LHC cascades down to the $\tilde{\chi}_{1}^{0}$ NLSP along with additional jets arising from the cascade. In situations where the strong sector is not kinematically accessible, it is worthwhile to explore signals from the direct production of the low-lying Higgsinos decaying promptly to the NLSP $\tilde{\chi}_{1}^{0}$, which then further decays to a Higgs/ $Z$ gauge boson and the $\tilde{G}$ LSP. As discussed in Sec. III A, such a scenario would lead to $h h / h Z / Z Z$ final states with/without extra hard jets arising from the strong sector cascade.

Motivated by the characteristics of a Higgsino NLSP spectrum, among the multifarious signatures possible, we focus on a final state consisting of a Higgs and $Z$ boson along with large $\mathbb{E}_{T}$ as the primary signature of such a scenario. In addition, to study the characteristic polarization of the $Z$ boson coming from the decay of the NLSP, we require an efficient and cleaner mode of reconstruction, which can only come through the leptonic decay of the weak gauge boson. Note that for the hadronic decays of a Higgs and $Z$ boson contributing to the signal rates, the corresponding SM hadronic background would also be significantly bigger. We therefore choose a final state that includes at least one $b$ jet and two same-flavor opposite sign leptons along with $\mathscr{E}_{T}$. Owing to the presence of leptons in the final state, this is a relatively clean channel to observe at LHC as compared to an all hadronic final state. Since the LSP is a very light $\tilde{G}$, the ensuing $h / Z$ from the NLSP decay and hence, the $b$ jets and/or leptons have large transverse momentum $\left(p_{T}\right)$, thereby leading to a large $\mathbb{E}_{T}$, where $\vec{E}_{T}=-\vec{p}_{T_{\text {vis }}}$ (balancing the net transverse momenta, $\vec{p}_{T_{\text {vis }}}$ of the visible particles). No specific criteria is imposed on the number of light jets in the scenario as will be present if the signal arises from the decay of the squarks or gluinos to the NLSP. This is because our choice of an inclusive final state signal would be able to highlight the presence of a Higgsino-like NLSP irrespective of the rest of the underlying MSSM spectrum, i.e., with/without the strong sector placed above the low-lying Higgsinos. 


\section{A. Signal, background, and event selection criteria}

We consider the following SUSY production processes involving squarks as well as the low-lying Higgsinos to be pair produced when kinematically accessible:

$$
p p \rightarrow \tilde{q}_{i} \tilde{q}_{j}, \tilde{q}_{i} \tilde{q}_{j}^{*}, \tilde{q}_{i}^{*} \tilde{q}_{j}^{*}, \tilde{q} \tilde{g}, \tilde{\chi}_{1}^{0} \tilde{\chi}_{2}^{0}, \tilde{\chi}_{1}^{ \pm} \tilde{\chi}_{1}^{0}, \tilde{\chi}_{1}^{ \pm} \tilde{\chi}_{2}^{0}, \tilde{\chi}_{1}^{+} \tilde{\chi}_{1}^{-} .
$$

Note that the gluinos are heavier compared to the squarks and Higgsinos, and their contribution to the signal is subdominant. When the signal is generated from the pair production of the strongly interacting sparticles, the final state consists of at least two hard jets in the $h h / h Z / Z Z$ final state along with a pair of invisible gravitinos, which contribute to the large $\mathbb{E}_{T}$. Among the possible combinations of the decay products of $h$ and $Z$, we primarily focus on the $\geq 1 b+\ell^{+} \ell^{-}$final state along with $\mathbb{E}_{T}$. Since the $Z$ decays leptonically, it gives a cleaner channel and better control over the SM backgrounds as compared to a hadronic final state.

We generate the signal events in MadGraph_v5 [45] using the model UFO files available from FeynRules [46]. Subsequently, parton level events are showered and hadronized using PYTHIA $[47,48]$, and a detector simulation is performed using DELPHES [49]. Jets (including $b$ jets) are reconstructed using the anti-kT algorithm [50], using FastJet [51] with minimum transverse momentum, $p_{T}>20 \mathrm{GeV}$ within a cone $\Delta R=0.4$. Charged leptons are reconstructed in a cone of $\Delta R=0.2$ with a maximum energy deposit in the cone from all other particles limited to $10 \%$ of the $p_{T}$ of the lepton. The significant contributions to the SM background for the given final state come from

(i) $t \bar{t},\left(t \rightarrow b W^{+}, W^{+} \rightarrow \ell^{+} \nu\right)$

(ii) $h Z+$ jets, $\left(h \rightarrow b \bar{b}, Z \rightarrow \ell^{+} \ell^{-}\right)$

(iii) $t \bar{t} Z,\left(Z \rightarrow \ell^{+} \ell^{-}\right)$

(iv) $Z Z,\left(Z \rightarrow b \bar{b}, Z \rightarrow \ell^{+} \ell^{-}\right)$

(v) $W^{ \pm} W^{\mp} Z,\left(Z \rightarrow \ell^{+} \ell^{-}\right)$

(vi) $l^{+} l^{-} b \bar{b}+\nu \bar{\nu}$.

Although the QCD background has a large cross section, it has a negligible contribution to the signal region characterized by large $\mathbb{E}_{T}$ as well as effective mass $\left(M_{\mathrm{Eff}}\right)$, which helps probe the heavy mass scale of the SUSY particles and would serve as an effective discriminator between the SUSY signal and SM background. For an SM background, we perform MLM matching [45] when needed with QCUT $=$ $20-30 \mathrm{GeV}$.

\section{B. Primary selection criteria}

We choose the following basic selection criteria to identify leptons $(e, \mu)$ and $b$ jets in the signal and background:

(i) The charged leptons are identified with $p_{T}>$ $10 \mathrm{GeV}$ and $|\eta|<2.5$.

(ii) All reconstructed $b$ jets have $p_{T}>30 \mathrm{GeV}$ and $|\eta|<2.5$.

(iii) Jets and leptons are isolated with $\Delta R_{i j}>0.4$ and $\Delta R_{\ell \ell}>0.2$.

\section{Signal analysis: At least $1 b-$ jet $+\ell^{+} \ell^{-}+\mathbb{E}_{T}$}

We note that for the signal, since the NLSP-LSP mass gap is large, the transverse momenta carried by the decay products are large thereby ensuring a large amount of $\boldsymbol{E}_{T}$ in the event. Figure 2 shows the normalized differential distribution of a few kinematic variables $\left(M_{\text {Eff }}\right.$ and $\left.\mathbb{E}_{T}\right)$ for BP1 and BP4 along with the background. The SUSY signal distributions for the missing transverse energy $\left(\mathscr{E}_{T}\right)$ and effective mass $\left(M_{\text {Eff }}\right)$ are widely separated from the SM background for BP4 in the presence of a heavy NLSP. However, the signal events peak at a much lower value of $M_{\mathrm{Eff}} \sim 850 \mathrm{GeV}$ for BP1, while significant events of the signal are found at large $M_{\text {Eff }}$ values $\sim 2.0 \mathrm{TeV}$ for BP4. Note that for BP4, this is due to the high transverse momentum of the jets and leptons arising from the decay cascades of the heavy $\mathcal{O}(2) \mathrm{TeV}$ range sparticles. However, for BP1 with a light NLSP, there is considerable overlap of the kinematic distributions with the background while differing in the tail of the distribution. This happens because the dominant contribution to the signal comes from the direct production of the light Higgsino sector as compared to the strong production cross section. We break our analysis in two parts to study different scenarios that can present themselves at LHC. The signal from a heavy
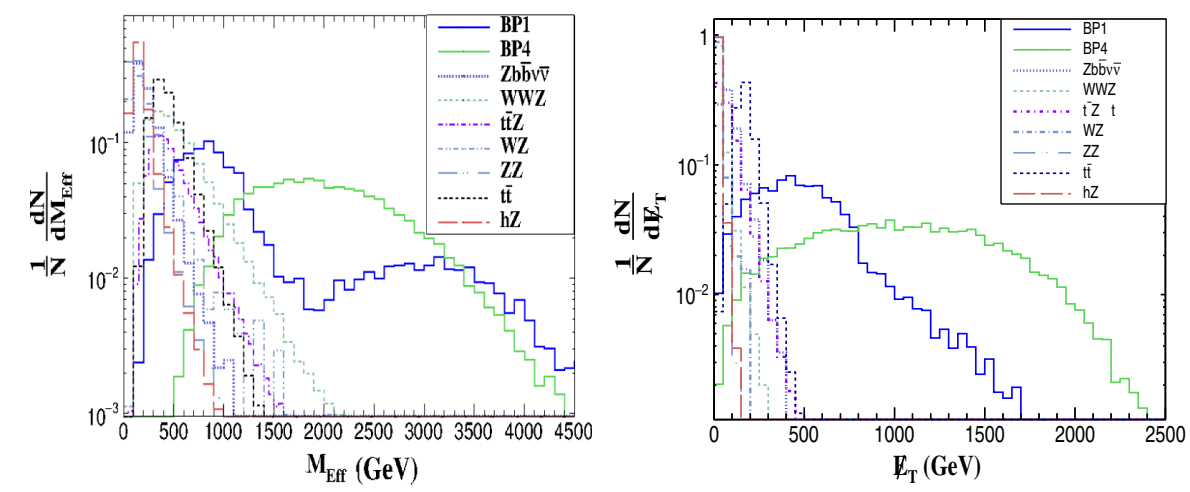

FIG. 2. Distribution of few useful kinematic variables before application of any selection cuts. 
spectrum of $\mathcal{O}(2) \mathrm{TeV}$, including the NLSP, that can only have a relevant signal contribution through the production of strongly interacting sparticles at the LHC is optimized using cuts in Analysis 1, while the signal for relatively lighter electroweakino states being directly accessible at LHC with smaller contributions from the strong sector is analyzed in Analysis 2. Appropriate cuts on the relevant kinematic variables will be crucial to remove SM background in the subsequent collider analyses to study the two scenarios discussed above.

\section{Analysis 1}

As a crucial part of our analysis is dependent on the reconstruction of the $Z$ boson in the events through the dilepton mode, the event rate for the signal will suffer due to the small branching fraction of the gauge boson to charged leptons. In addition, if we intend to reconstruct the light Higgs boson too using double $b$-tag jets, we will end up restricting our search sensitivity significantly. We therefore need to select events using proper cuts to be able to identify the $Z$ boson as well as imply a Higgs like event. In order to select such a final state, we implement the following event selection criterion to retain a significant amount of signal against the SM background:

(i) D1: We select a final state with two opposite sign leptons of the same flavor $\left(N_{\ell}=2\right.$ with $\left.p_{T}>20 \mathrm{GeV}\right)$ and at least one $b$ jet with $p_{T}>30 \mathrm{GeV}$.

(ii) D2: To reconstruct the $Z$ boson, we demand that the invariant mass of a dilepton pair (opposite sign, same flavor) in the signal events is within the $Z$ mass window satisfying $76<M_{\ell^{+} \ell^{-}}<106 \mathrm{GeV}$.

Another kinematic variable of importance is the stransverse mass $M_{T_{2}}$ [52]. It is reconstructed using the $p_{T}$ of the charged lepton pair along with $\mathbb{E}_{T}$. For SM processes such as $t \bar{t}, M_{T_{2}}$ shows an end point value $\sim M_{W}$. For SUSY processes, the end point is determined by the mass difference between the NLSP and LSP. For a light keV scale LSP and TeV NLSP, the end point is large compared to $M_{W}$ and can serve as an effective discriminator between SUSY signals and the dominant SM background subprocesses.

(i) D3: We demand a cut on the kinematic variable $M_{T_{2}}>90 \mathrm{GeV}$ to remove backgrounds from $t \bar{t}$.

The other important kinematic variable is the effective mass, $\quad M_{\text {Eff }}=p_{T}\left(\ell^{+}\right)+p_{T}\left(\ell^{-}\right)+\mathbb{E}_{T}+\sum p_{T_{i}}(j)$, the scalar sum of the transverse momenta of the visible jets, leptons, and $\mathscr{E}_{T}$ in the event. $M_{\mathrm{Eff}}$ reflects the mass scale of the heavy SUSY particles and hence, is an efficient variable to suppress SM background. However, the choice of a strong cut on $M_{\text {Eff }}$ would choose to retain contributions from a very heavy spectrum, and therefore, in this case, we focus on the benchmarks that contribute mainly through the production of the strong sector sparticles, viz. BP4 and BP5. Note that the strong sector for BP1 is of similar value to BP4 and BP5, and therefore, the signal rates coming from the strong sector would be very similar. However, a dominant fraction of signal events would come from the light Higgsino production, and the cuts given below are not particularly optimized to study BP1. The BP3 signal, on the other hand, becomes very small. We shall discuss the BP1 and BP3 benchmarks separately in Analysis 2. Additional cuts on the events are

(i) D4: Since nearly all the SUSY particles excluding the LSP are heavy for BP4 and BP5, a large $M_{\mathrm{Eff}}$ is expected for the signal over the SM background as shown in Fig. 2. We therefore demand a strong cut of $M_{\text {Eff }}>2 \mathrm{TeV}$. This cut renders the signal for other benchmarks to a relatively smaller value.

(ii) D5: In addition, we also put a strong cut on missing transverse energy, $\mathbb{E}_{T}>300 \mathrm{GeV}$ to further remove remaining contributions from SM background processes.

We show the cut-flow result of our analysis for the signal and SM background in Table IV. As expected, the signal rates coming from a $2 \mathrm{TeV}$ squark sector yields quite small numbers, even with an integrated luminosity of $3000 \mathrm{fb}^{-1}$. The overwhelmingly huge SM background is brought in control by primarily using the $M_{T_{2}}$ cut and is then rendered negligibly small using the combination of $M_{\text {Eff }}$ and $\mathbb{E}_{T}$ cuts. We find that the sequence of cuts shown in Table IV affects the signal slightly with a suppression of the signal rate of less than $50 \%$ for BP5. Thus, we find a significant number of SUSY signal events surviving the event selection. Note that while a $\sim 75 \%$ suppression of signal events happen for BP1, it is still quite large compared to the SM background, unlike that for BP3.

TABLE IV. Number of signal and background events for $\geq$ $1 b+\ell^{+} \ell^{-}+\mathbb{E}_{T}$ at $\sqrt{s}=13 \mathrm{TeV}$ LHC for $\mathcal{L}=3000 \mathrm{fb}^{-1}$ using cuts D1 - D5. Note that the total number of background events have been rounded off to the nearest integer. Cross sections for SUSY signals have been scaled using NLO K factors [53] and wherever available, NLO + NLL K factors [54]. Cross sections for SM background processes have been scaled using NLO K factors [45] and wherever available, NNLO K factors [55-59] have been used.

\begin{tabular}{lccccc}
\hline \hline Signal & D1 & D2 & D3 & D4 & D5 \\
\hline BP1 & 130 & 104 & 91 & 39 & 33 \\
BP3 & 98 & 83 & 74 & 2 & 2 \\
BP4 & 22 & 17 & 17 & 16 & 16 \\
BP5 & 62 & 33 & 30 & 28 & 26 \\
SM background & $\mathbf{D 1}$ & $\mathbf{D 2}$ & $\mathbf{D 3}$ & $\mathbf{D 4}$ & $\mathbf{D 5}$ \\
$t \bar{t}$ & 365125 & 64968 & 186 & $\ldots$ & $\ldots$ \\
$h Z$ & 29348 & 28360 & 781 & 1.76 & 0.16 \\
$Z Z$ & 178581 & 172636 & 2124 & 15 & 2.3 \\
$t \bar{t} Z$ & 3043.3 & 2111 & 287 & 6.14 & 0.98 \\
$t \bar{t} W$ & 9121 & 1802 & 14 & $\ldots$ & $\ldots$ \\
$W W Z$ & 159 & 153 & 13 & 0.65 & 0.074 \\
Total background & & & & & 3 \\
\hline \hline
\end{tabular}


TABLE V. Required luminosities for observing the SUSY signal for the different benchmarks at $\sqrt{s}=13 \mathrm{TeV}$ LHC run. The numbers in the parentheses include $10 \%$ systematic errors in the background.

\begin{tabular}{lcc}
\hline \hline Benchmark & $\begin{array}{c}\mathcal{L}\left(\text { in } \mathrm{fb}^{-1}\right) \\
\text { for } 3 \sigma \text { excess }\end{array}$ & $\begin{array}{c}\mathcal{L}\left(\text { in } \mathrm{fb}^{-1}\right) \\
\text { for } 5 \sigma \text { excess }\end{array}$ \\
\hline BP1 & $240(250)$ & $665(695)$ \\
BP4 & $1112(1178)$ & $3090(3270)$ \\
BP5 & $340(356)$ & $943(988)$ \\
\hline \hline
\end{tabular}

We compute the statistical significance $(\mathcal{S})$ of the above signals using the formula in Eq. (14) and show the required integrated luminosities to observe and discover the signal in Table V,

$$
\mathcal{S}=\sqrt{2 \times\left[(s+b) \ln \left(1+\frac{s}{b}\right)-s\right]},
$$

where $s$ and $b$ refer to the number of signal and background events, respectively. We observe that benchmarks BP4 and BP5 require large integrated luminosities, whereas BP3 with a decoupled squark sector is out of reach of LHC. Although BP1 is observable at LHC, the large $M_{\text {Eff }}$ cut reduces the contribution from the light Higgsino sector, which is directly accessible at LHC. Therefore, this analysis is more sensitive to the case of heavier spectra that also includes the NLSP to be quite heavy, such as BP4 and BP5. However, with a light Higgsino sector and similar squark masses to BP4, such as in BP1, we are still able to get a relatively healthy number for the signal albeit after losing a large part of the signal events. A more optimized set of cuts is used in Analysis 2 to study the scenario with lighter NLSP mass.

Let us also comment on the prospect of multijet searches as discovery channels for our scenario. Using the SM backgrounds of the multijet analyses [36], we estimate the reach of the squark masses to be $2.78 \mathrm{TeV}$ to achieve a $5 \sigma$ discovery at an integrated luminosity of $3000 \mathrm{fb}^{-1}$ at LHC. For such a heavy spectrum, the final state channel of $\geq$ $1 b+\ell^{+} \ell^{-}+\mathbb{E}_{T}$ would not be within the LHC reach, and therefore, the multijet channel would be the best discovery channel.

\section{E. Analysis 2}

We now focus on the signal contribution arising dominantly from the electroweak sector of sparticles with/ without the strong sector, as in benchmark BP1 and BP3. Since the Higgsino sector is lighter, a strong cut on $M_{\text {Eff }}$ as used in D4 will deplete the signal significantly in this case. Therefore, we employ a different set of cuts to investigate the signal region $\left(\geq 1 b+\ell^{+} \ell^{-}+\mathbb{E}_{T}\right)$ arising from the low-lying Higgsino sector. We consider the contributions from the Higgsino sector in addition to the strong sector for the benchmarks in our study when they are kinematically accessible and study the benchmarks BP1 and BP3. As the final state remains unchanged, the cuts implemented on both signal and background in Analysis 1 would still be useful for background suppression. The implemented cuts therefore remain similar except for the excluded $M_{\text {Eff }}$ cut, optimized for the two benchmarks

(i) E1: As in Analysis 1, we select a final state with two opposite sign leptons of same flavor $\left(N_{\ell}=2\right.$ with $p_{T}>20 \mathrm{GeV}$ ) and at least one $b$ jet with $p_{T}>30 \mathrm{GeV}$.

(ii) E2: To reconstruct the $Z$ boson, we demand that the invariant mass of the dilepton pair in the signal events is within the $Z$ mass window, satisfying $76<M_{\ell^{+} \ell^{-}}<106 \mathrm{GeV}$.

(iii) E3: As before, $M_{T_{2}}$ is an efficient cut to reduce background contributions from $t \bar{t}$ to the signal region. We demand a slightly stronger cut of $M_{T_{2}}>$ $120 \mathrm{GeV}$ in this case as it helps improve the signalto-background ratio.

(iv) E4: The SUSY signal has a larger $\mathbb{E}_{T}$ as compared to the SM background. Hence, $\mathbb{E}_{T}>300 \mathrm{GeV}$ cut

TABLE VI. Number of signal and background events for $\geq$ $1 b+\ell^{+} \ell^{-}+\mathscr{E}_{T}$ at $\sqrt{s}=13 \mathrm{TeV}$ LHC for $\mathcal{L}=3000 \mathrm{fb}^{-1}$ using cuts $\mathbf{E} 1-\mathbf{E} 4$. Note that the total number of background events have been rounded off to the nearest integer. Cross sections for SUSY signals have been scaled using NLO K factors [53] and wherever available, NLO + NLL K factors [54]. Cross sections for SM background processes have been scaled using NLO K factors [45] and wherever available, NNLO K factors [55-59] have been used.

\begin{tabular}{lcccc}
\hline \hline BP1 & $\mathbf{E 1}$ & $\mathbf{E 2}$ & $\mathbf{E 3}$ & $\mathbf{E 4}$ \\
\hline$\tilde{\chi}_{1}^{ \pm} \tilde{\chi}_{1}^{ \pm}$ & 16 & 13 & 11 & 9 \\
$\tilde{\chi}_{1}^{ \pm} \tilde{\chi}_{1 / 2}^{0}$ & 65 & 54 & 47 & 36 \\
$\tilde{\chi}_{2}^{0} \tilde{\chi}_{1}^{0}$ & 16 & 14 & 12 & 9 \\
$\tilde{q} \tilde{q}, \tilde{q} \tilde{g}$ & 47 & 36 & 28 & 26 \\
Total & & & & 80 \\
$\mathbf{B P 3}$ & $\mathbf{E 1}$ & $\mathbf{E 2}$ & $\mathbf{E 3}$ & $\mathbf{E 4}$ \\
$\tilde{\chi}_{1}^{ \pm} \tilde{\chi}_{1}^{ \pm}$ & 33 & 27 & 24 & 18 \\
$\tilde{\chi}_{1}^{ \pm} \tilde{\chi}_{1 / 2}^{0}$ & 126 & 107 & 87 & 65 \\
$\tilde{\chi}_{2}^{0} \tilde{\chi}_{1}^{0}$ & 33 & 28 & 24 & 18 \\
Total & & & & \\
SM background & $\mathbf{E 1}$ & $\mathbf{E 2}$ & $\mathbf{E 3}$ & $\mathbf{E 4}$ \\
$t \bar{t}$ & 365125 & 64968 & $\ldots$ & $\ldots$ \\
$h Z$ & 29348 & 28360 & 298 & 0.67 \\
$Z Z$ & 178581 & 172636 & 774 & 6.61 \\
$t \bar{t} Z$ & 3043 & 2111 & 151 & 8.6 \\
$t \bar{t} W$ & 9121 & 1802 & 1 & $\ldots$ \\
$W W Z$ & 159 & 153 & 6 & 0.23 \\
$l^{+} l^{-} b \bar{b}+\mathbb{E}_{T}$ & 2933 & 2905 & 312 & 34.7 \\
Total & & & & 51 \\
\hline \hline
\end{tabular}


TABLE VII. Required luminosities for observing the SUSY signal for the different benchmarks at $\sqrt{s}=13 \mathrm{TeV}$ LHC run. The numbers in the parentheses include $10 \%$ systematic errors in the background.

\begin{tabular}{lcc}
\hline \hline Benchmark & $\begin{array}{c}\mathcal{L}\left(\text { in } \mathrm{fb}^{-1}\right) \\
\text { for } 3 \sigma \text { excess }\end{array}$ & $\begin{array}{c}\mathcal{L}\left(\text { in } \mathrm{fb}^{-1}\right) \\
\text { for } 5 \sigma \text { excess }\end{array}$ \\
\hline BP1 & $310(333)$ & $862(924)$ \\
BP3 & $208(226)$ & $577(626)$ \\
\hline \hline
\end{tabular}

helps reduce a significant part of the remnant contributions from SM background.

The cut-flow table for the signal and SM background are as shown in Table VI. We rely on a slightly stronger cut on $M_{T_{2}}$ to ensure substantial removal of the $t \bar{t}$ background while retaining the signal events. However, other background contributions, such as that from $l^{+} l^{-} b \bar{b}+\mathbb{E}_{T}$, remain. This still gives a significantly large event rate for the signal as compared to Analysis 1 , and thereby allowing a $\sim(8-10) \sigma$ discovery possible with $\mathcal{L}=3000 \mathrm{fb}^{-1}$. Since both the benchmarks have similar branching fractions into the $Z$ and Higgs mode, the difference in the required integrated luminosity is due to the fact that the NLSP mass is heavier in BP1 than in BP3. The required luminosity for observing a $3 \sigma$ and $5 \sigma$ significance at LHC are shown Table VII. We conclude that both BP1 and BP3 are well within the discovery reach of the high luminosity run of LHC.

We are now set to study the efficacy of the signal that we have analyzed to identify the nature of the NLSP and its inherent composition with respect to the gaugino-Higgsino admixture in the following section.

\section{A DISTINGUISHING FEATURE: LONGITUDINAL VS TRANSVERSE GAUGE BOSONS}

We are interested in a Higgsino-like lightest neutralino $\tilde{\chi}_{1}^{0}$ for which $\tilde{\chi}_{1}^{0} \rightarrow h \tilde{G}$ is an obvious decay channel. In addition, the Higgsino also couples to the imaginary parts of the neutral component of the two Higgs doublets. This leads to Higgsino-goldstone-gravitino interactions. The goldstone, on the other hand, approximates the longitudinal component of the $Z$, when the relevant energy scale is much larger than $m_{Z}$. Thus, for a Higgsino-like $\tilde{\chi}_{1}^{0}$ with $m_{\tilde{\chi}_{1}^{0}} \gg m_{Z}$, one also expects the decay $\tilde{\chi}_{1}^{0} \rightarrow Z \tilde{G}$, when the $Z$ is longitudinal.

Although our focus is on the polarization of $Z$ boson in the context of SUSY in this work, the polarization information of vector bosons may be extremely useful even for non-SUSY scenarios, where a polarized gauge boson is likely to be produced from the decay of a heavy particle. Thus, the features of the longitudinal $Z$ boson, which will be discussed in detail in this work, are also applicable for other scenarios as well. For example, the presence of longitudinal gauge bosons from heavy Higgs decays have been studied in earlier works in the context of the Tevatron [60]. LHC analyses have also looked at features of longitudinal gauge bosons in the SM [61]. In case an excess over SM is observed, it is of crucial importance to extend current search strategies to characterize BSM scenarios by studying variables sensitive to the polarization information of the gauge bosons via their decay products. Although there have been several studies in the context of $e^{+} e^{-}$colliders focusing on studies of polarizations of the incoming electron-positron beams or polarization of the final state particles, there are few analogous studies with respect to the LHC utilizing these techniques [62]. The polarization of a $Z$ boson has been studied briefly in [62] with respect to the LHC in a similar scenario however in displaced dilepton final states arising from the $Z$ boson decay using the angular variable $\cos \theta^{*}$ discussed below. We discuss analytically some basic variables found in the literature, which distinguish longitudinal and transverse gauge bosons. The differential decay rates for the transversely polarized and longitudinally polarized $Z$ boson in the rest frame of $Z$ boson are [60]

$$
\begin{gathered}
\frac{d \Gamma_{T}}{d \cos \theta^{*}} \propto\left(1 \pm \cos \theta^{*}\right)^{2} \\
\frac{d \Gamma_{L}}{d \cos \theta^{*}} \propto \sin ^{2} \theta^{*},
\end{gathered}
$$

where $\Gamma_{T}=\Gamma\left(\tilde{\chi}_{1}^{0} \rightarrow Z_{T} \tilde{G}\right)$ and $\Gamma_{L}=\Gamma\left(\tilde{\chi}_{1}^{0} \rightarrow Z_{L} \tilde{G}\right)$ are the partial decay widths of the $\tilde{\chi}_{1}^{0}$ to a transverse $Z$ boson $\left(Z_{T}\right)$ and longitudinal $Z\left(Z_{L}\right)$ boson, respectively. The angle $\theta^{*}$ is defined as the angle the outgoing lepton (arising from the $Z$ boson decay) makes with the $Z$ boson in its rest frame with the reference direction being the boost direction of the $Z$ boson in the laboratory frame. The dependence of the decay width; i.e., $\left(1 \pm \cos \theta^{*}\right)^{2}$ corresponds to $k=\mp 1$ state and $\sin ^{2} \theta^{*}$ corresponds to $k=0$ state, where $k$ is the helicity of the $Z$ boson. To highlight the difference, we choose the NLSP from a few of our benchmarks and generate a normalized distribution for $\cos \theta^{*}$, where the NLSP is decaying at rest and gives the $Z$ boson as its decay product. The simple illustration of this reconstruction is shown in Fig. 3, where BP1 represents a dominantly Higgsino-like NLSP, BP2 represents a dominantly gauginolike NLSP, while BP4 represents a comparable admixture of gauginos and Higgsinos in the NLSP.

We now go ahead and consider the full analysis for the signal $\geq 1 b+\ell^{+} \ell^{-}+\mathbb{E}_{T}$ and focus on the negatively charged lepton. Note that we expect distributions in $\cos \theta^{*}$, as highlighted in Fig. 3, for the $Z$ polarization to be robust against the energy smearings in the detector and the full detector simulation. To show this, we compare both parton-level analysis to the signal events obtained after detector simulations. We plot the normalized distributions 


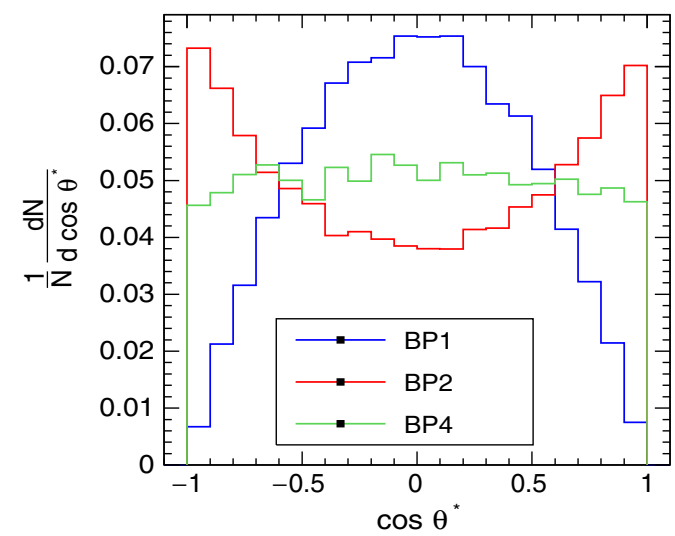

FIG. 3. Normalized distribution of $\cos \theta^{*}$ of the negatively charged lepton $\left(\ell^{-}\right)$arising from the $\tilde{\chi}_{1}^{0}$ NLSP decay at rest corresponding to the benchmarks BP1, BP2, and BP4 with the isolation variable $\Delta R>0.2$ for the leptons.

for $\cos \theta^{*}$ of the negatively charged daughter lepton of the $Z$ boson in Fig. 4 at the parton level (left) and detector level (right-panel) for our benchmarks BP1, BP2, and BP4, where one NLSP decays to a $Z$ boson along with a $\tilde{G}$. Recall, BP1 has a purely Higgsino-like NLSP ( 99\%), BP2 with purely gauginolike NLSP $(\sim 100 \%)$, and BP4 has $\sim 31 \%$ gaugino admixture in the NLSP. We observe in Fig. 4 that the distributions for the negatively charged lepton (for BP1 and BP2) are largely similar at both parton and detector level simulations, to the expected distributions as shown in Fig. 3. ${ }^{4}$ For BP4, where the NLSP is a more democratic superposition of the gaugino and Higgsino states, a slightly flat and broad peak for $\cos \theta^{*}$ is observed. In addition, the NLSP mass is around $2 \mathrm{TeV}$, which results in a very boosted $Z$ boson in the final state. The event selection criteria can in principle have adverse effects in this case and modify the distributions. The most notable effect for BP4 is that the distribution starts to resemble features similar to the gauginolike NLSP (BP2) at both parton and detector-level simulations. This we find is due to the fact that when the $Z$ boson is highly boosted, the pair of charged leptons coming from the $Z$ boson decay get more collimated with a very small opening angle. This in turn would mean that a larger isolation requirement for the charged leptons would lead to loss of events and also affect the $\cos \theta^{*}$ distribution.

In our analysis, we have used the default DELPHES card using a small cone radius $R=0.2$ and a maximum energy deposit in the cone being $10 \%$ of the $p_{T}$ of the lepton. A lepton-lepton isolation cut on $\Delta R>0.2$ reduces the peak of the $\cos \theta^{*}$ plot. To counter the consequent reduction in signal events, for BP4, a relatively relaxed lepton identification criterion can be useful for our purpose. To highlight this, we identify the charged leptons with a much larger

\footnotetext{
${ }^{4}$ Similar distributions for $\cos \theta^{*}$ are expected for the positively charged leptons.
}

cone radius of $R=0.5$ for lepton identification and also demand that a large energy deposit with respect to the $p_{T}$ of the lepton is allowed in the cone ( $\sim 12 \%$ for electrons and $25 \%$ for muons). The distribution still retains the gauginolike behavior for an isolation of $\Delta R>0.2$ as in the parton level but starts agreeing with the Higgsino-like feature (as in the parton-level case) when the separation between the charged leptons is chosen to be loose with $\Delta R>0.05$ or $\Delta R>0.1$ as can be seen in the bottom-right panel of Fig. 4 .

The qualitative differences observed in the distributions of the negatively charged lepton as the gaugino admixture increases in the NLSP amongst the three cases may be effectively captured by defining asymmetry variables in $\cos \theta^{*}$, which could clearly discriminate between a longitudinal and transverse $Z$ boson. Taking a cue from the features of $\cos \theta^{*}$, we construct a variable which highlights this difference through an asymmetry amongst the observed $\cos \theta^{*}$ values for the Higgsino-like and gauginolike NLSP. The asymmetry variable, $C_{\theta_{\mathrm{Z}}}$, as defined in Eq. (17), serves to enhance the features of the longitudinally polarized $Z$ in comparison to the transversely polarized $Z$, such that they would be less affected if detector simulation effects smear the polarization dependence of the angular or energy observables. We define

$$
C_{\theta_{Z}}=\frac{N_{A}-N_{B}-N_{C}}{N_{A}+N_{B}+N_{C}}
$$

where $N_{I}$ 's stand for events whereas the subscript $I=A, B, C$ represent the angular regions in $\theta^{*}$ given by $A=[\pi / 3,2 \pi / 3]$, $B=[0, \pi / 3]$, and $C=[2 \pi / 3, \pi]$. The numerator focuses only on the asymmetry features while the denominator is the total number of events for $-1<\cos \theta^{*}<1$. Based on the construction of $C_{\theta_{Z}}$, a positive value is indicative of a Higgsino-like NLSP whereas negative values indicate a gauginolike NLSP. Since $C_{\theta_{Z}}$ is the normalized difference in the number of events corresponding to $\left|\cos \theta^{*}\right|<0.5$ and $\left|\cos \theta^{*}\right|>0.5$, a Higgsino-like NLSP gives larger events around $\cos \theta^{*}=0$ as $N_{A}>\left(N_{B}+N_{C}\right)$, whereas for the gauginolike NLSP, the distribution peaks around $\cos \theta^{*} \sim$ \pm 0.8 i.e., $\left(N_{B}+N_{C}\right)>N_{A}$. Therefore, the latter shows a negative sign as compared to the former. We list the values of $C_{\theta_{Z}}$ for cases when the NLSP decays at rest $\left(C_{\theta_{Z}}^{\text {rest }}\right)$ and compare this with parton-level $\left(C_{\theta_{Z}}^{\text {parton }}\right)$ results and full detector-level simulation $\left(C_{\theta_{Z}}\right)$ of our Analysis 1 in Table VIII, for the benchmarks BP1 and BP2 (which correspond to an almost pure Higgsino and pure gaugino compositions, respectively). ${ }^{5}$

Note that the values of $C_{\theta_{Z}}$ are in good agreement with the parton level $C_{\theta_{Z}}^{\text {parton }}$ results. For the pure Higgsino-like NLSP

\footnotetext{
${ }^{5}$ The results in Table VIII are produced by using only squark pair production. However, the generic feature remains unchanged even when all production modes are included.
} 

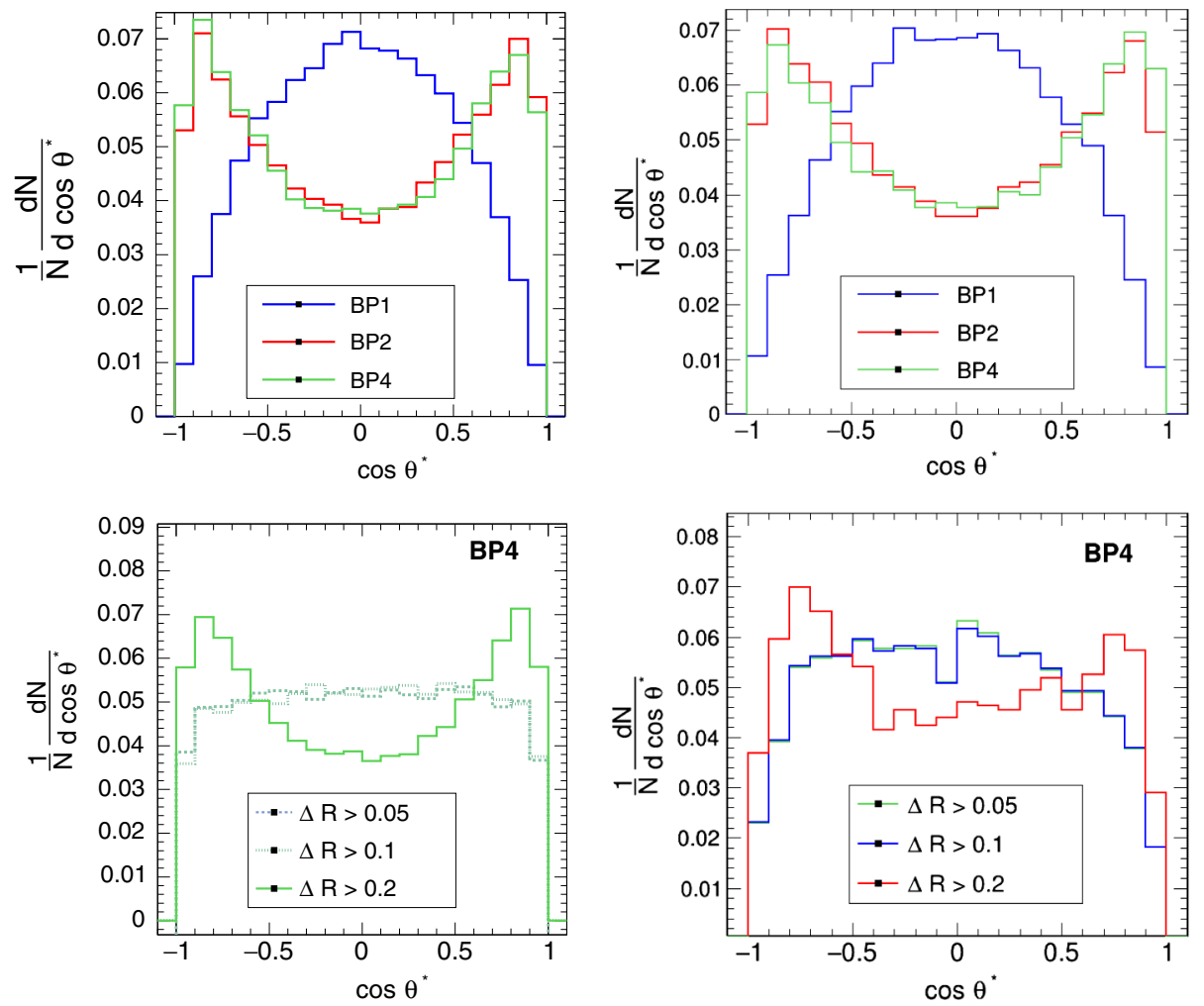

FIG. 4. Normalized distribution of $\cos \theta^{*}$ of the negatively charged lepton $\left(\ell^{-}\right)$arising from the $\tilde{\chi}_{1}^{0}$ NLSP at the parton level (top left panel) and after detector simulation (top right panel) using Analysis 1, corresponding to the benchmarks BP1, BP2, and BP4. In the bottom panel, we present the plots for BP4 at the parton level (left) and at the detector level (right) for various $\Delta R$ values as discussed in the text.

(BP1), $C_{\theta_{Z}}$ is large and positive, whereas the pure gauginolike NLSP (BP2) shows a negative value. We find that the $C_{\theta_{Z}}$ value starts decreasing as the gaugino admixture in the NLSP is increased when compared to BP1. Thus, with increasing gaugino admixture the asymmetry value becomes negative as shown for BP2. The most notable change is observed for the BP4 with an intermediate gauginoHiggsino admixture. $C_{\theta_{Z}}$ value is $\sim 0.021$ when the NLSP decays at rest with the small positive value still hinting at a larger Higgsino admixture. However, it turns negative for the analysis where the NLSP appears from cascade decays of the squark, both at the parton and the detector level owing largely to the effect of isolation cuts and detector smearing effects, which modify the $\cos \theta^{*}$ distribution as seen in Fig. 4 and discussed earlier. We note that the $C_{\theta_{z}}^{\text {parton }}$ value becomes positive giving $C_{\theta_{Z}}^{\text {parton }}=0.04,0.05$ for the loose isolation requirement and identification of the charged lepton with $\Delta R>0.05,0.1$ as against $C_{\theta_{Z}}^{\text {parton }}=-0.214$ for the tighter isolation cut of $\Delta R>0.2$. We expect that the same would be true when the events are passed through detector simulations, which would be consistent with observations made in the lower panels of Fig. 4.

An additional kinematic feature that can be used to study the polarization of the $Z$ boson, which in effect highlights the composition of the NLSP, is the charged lepton energy. Among others, the ratio of the energy carried by the charged lepton and antilepton also show a dependence on the polarization of the $Z$ boson with an energy $E$, via dependence on the angle $\theta^{*}$. The energy $\left(E_{\ell}\right)$ of the leptons in the laboratory frame [60] follows

$$
E_{\ell} \propto \frac{E}{2}\left(1 \pm \beta \cos \theta^{*}\right)
$$

TABLE VIII. Variation of the asymmetry variables $C_{\theta_{z}}^{\text {rest }}, C_{\theta_{Z}}^{\text {parton }}, C_{\theta_{Z}}$, and $C_{Z}$ as defined in the text, at the parton level and detector level after cuts D1 - D5 for benchmarks BP1 and BP2. The numbers for $C_{\theta_{Z}}$ and $C_{Z}$ include both signal and background in the computation of the observables and its associated $1 \sigma$ error for $\mathcal{L}=3000 \mathrm{fb}^{-1}$.

\begin{tabular}{lcccrrrr}
\hline \hline Benchmark & $m_{\tilde{\chi}_{1}^{0}}(\mathrm{GeV})$ & Higgsino admixture $(\%)$ & Gaugino admixture $(\%)$ & \multicolumn{1}{c}{$C_{\theta_{Z}}^{\text {rest }}$} & \multicolumn{1}{c}{$C_{\theta_{Z}}^{\text {parton }}$} & \multicolumn{1}{c}{$C_{\theta_{Z}}$} & \multicolumn{1}{c}{$C_{Z}$} \\
\hline BP1 & 810.9 & 99.83 & 0.17 & 0.38 & 0.33 & $0.35 \pm 0.16$ & $0.38 \pm 0.16$ \\
BP2 & 797.9 & 0.05 & 99.95 & -0.18 & -0.08 & $-0.05 \pm 0.21$ & $-0.02 \pm 0.21$ \\
\hline \hline
\end{tabular}



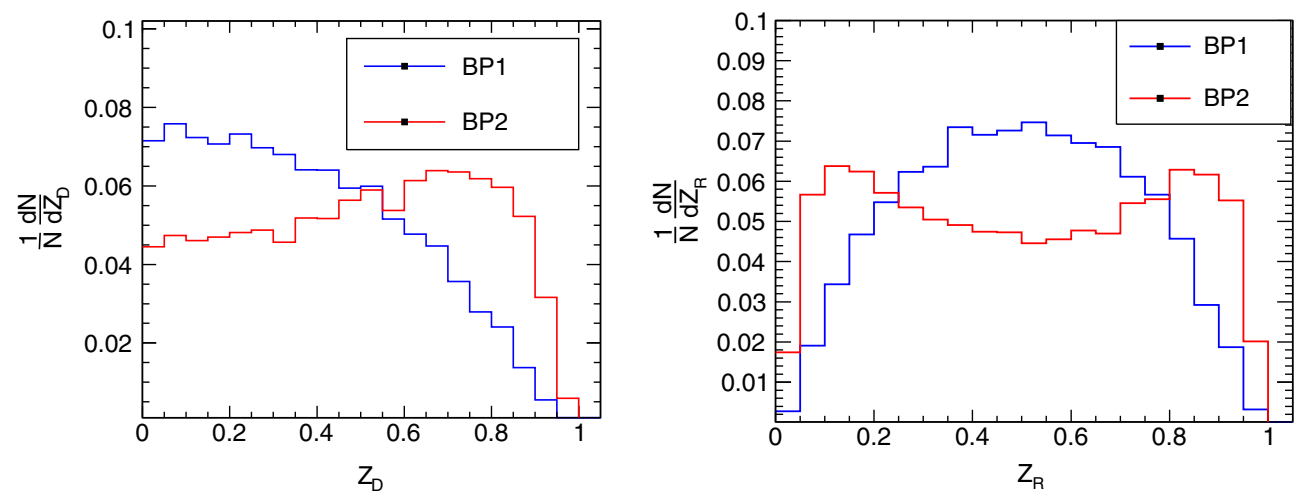

FIG. 5. Normalized distributions of the kinematic variables $Z_{D}$ and $Z_{R}$ as defined in the text for distinguishing between a Higgsino and gauginolike $\tilde{\chi}_{1}^{0}$ NLSP before cuts D1 - D5 The variables are as defined in the text. Here, we have plotted the observables for the process $\tilde{q} \tilde{q}$ with one of the squarks decaying as: $\tilde{q} \rightarrow q \tilde{\chi}_{1}^{0} \rightarrow q Z \tilde{G}, Z \rightarrow \ell^{+} \ell^{-}$.

Using this, we define two kinematic observables $Z_{D}$ and $Z_{R}$ (variations of such observables have been pointed out in earlier papers $[63,64]$ using jet substructure to study hadronic final states),

$$
Z_{D}=\frac{\left|E_{\ell^{-}}-E_{\ell^{+}}\right|}{E_{\ell^{-}}+E_{\ell^{+}}} ; \quad Z_{R}=\frac{E_{\ell^{-}}}{E_{\ell^{-}}+E_{\ell^{+}}} .
$$

We study the usefulness of these observables using simple cuts on kinematic variables after detector simulation effects are taken into account. For a predominantly longitudinal $Z$ boson, there is an equal sharing of energy of the parent among its daughter particles whereas for a transverse $Z$ boson, the energy sharing is unequal. The asymmetry is evident in Fig. 5, where the Higgsino-like NLSP peaks at $Z_{D}=0.1$ as compared to $Z_{D}=0.8$ for the gauginolike case. Similar effects are observed in the variable $Z_{R}$, which denotes the fraction of net leptonic energy carried away by the negatively charged lepton. The ratio peaks at $Z_{R} \simeq 0.5$ for BP1 as compared to $Z_{R} \simeq 0.1$ and $Z_{R} \simeq 0.8$ for the BP2, since for the former case, the leptons mostly have equal energy sharing, whereas unequal energy sharing occurs for the latter case. We define an asymmetry variable similar to $C_{\theta_{Z}}$, now referred to as $C_{Z}$ to capture the asymmetry in the values of $Z_{D}$ at the detector level,

$$
C_{Z}=\frac{N_{A}-N_{B}}{N_{A}+N_{B}}
$$

where $N_{A}$ refers to the number of events for $Z_{D}<0.5$ and $N_{B}$ represents events for $Z_{D}>0.5$, respectively. We list the $C_{Z}$ values in Table VIII and observe that $C_{Z}$ is positive for the Higgsino-like NLSP and negative for gauginolike NLSP. Note that the effect observed for the highly boosted $Z$ boson in $C_{\theta_{Z}}$ also shows up for $C_{Z}$ highlighting the consistency and importance of the isolation of the charged leptons. The statistical uncertainty in the observed asymmetry has been shown in Table VIII and is calculated using [65]

$$
\sigma(C)=\frac{\sqrt{1-C^{2}}}{\sqrt{N}},
$$

where $C=C_{\theta_{Z}}, C_{Z}$, while $N$ represents the total number of events. The uncertainty goes down as $1 / \sqrt{N}$, which means that a larger luminosity would help in improving the statistical significance; therefore, we emphasize that the distribution of $\cos \theta^{*}$ arising from the $Z$ boson decay as well as the associated asymmetry variables, $C_{\theta_{Z}}$ and $C_{Z}$ prove quite useful in identifying the nature of the NLSP. The distinctive features of the variables discussed for distinguishing a longitudinal and transversely polarized $Z$ boson are also applicable for new physics scenarios, where a polarized gauge boson is likely to be produced and therefore, can prove very important in studying BSM physics.

\section{SUMMARY AND CONCLUSIONS}

In this work, we have considered Higgsino-like NLSP in the presence of a light $\mathrm{keV} \tilde{G}$ LSP in the framework of phenomenological MSSM. The keV scale $\tilde{G}$ serves as a warm dark matter candidate, significantly relaxing constraints from dark matter searches on the MSSM spectrum and thereby, allowing low $\mu$ parameter values. In addition, presence of a light $\tilde{G}$ allows decay of the NLSP to a Higgs $/ Z$ boson and $\tilde{G}$ leading to hard $b$ jets and charged leptons in the final state along with large $\mathbb{E}_{T}$ carried away by the $\tilde{G}$. Such a scenario has been extensively explored by experiments, including the LHC, with a primary focus on the low-lying electroweak sector, leading to stringent constraints on the parameter space. The question that one ventures to answer in this study is as follows: What are the future prospects of detecting a Higgsino-like $\tilde{\chi}_{1}^{0}$ NLSP at LHC? If detected, how can we ascertain the nature of the NLSP?

We address this question by studying a specific final state: $\geq 1 b+\ell^{+} \ell^{-}+\mathbb{E}_{T}$ at $\sqrt{s}=13 \mathrm{TeV}$ motivated by the presence of at least one $b$ jet from the Higgs boson and 
an opposite sign same-flavor lepton pair from the $Z$ boson decay besides large $\mathbb{E}_{T}$. We choose a few representative benchmark points encompassing a light and heavy Higgsino sector with/without strong sector sparticles within the reach of LHC. We find that such a signal is discoverable in the upcoming runs of the high luminosity LHC after suitable cuts are applied. It is important to emphasize that such a semileptonic channel will prove crucial in identifying the nature of the NLSP, being relatively clean compared to an all hadronic final state, which may have a better discovery prospect. Thus, simultaneous use of both channels could be advocated for the purpose of discovery and identifying the nature of the NLSP. We focus on the presence of a dominantly longitudinal $Z$ boson arising from the decay of a Higgsino-like NLSP owing to the presence of the Goldstone boson as the longitudinal mode of $Z$ after electroweak symmetry breaking. This is quite a striking identification criteria if observable, for a Higgsinolike NLSP in sharp contrast to a dominantly gauginolike NLSP, which would dominantly decay to a transversely polarized $Z$. It is thus important to characterize the features of the longitudinally polarized $Z$ boson to ascertain the composition of the parent NLSP. The effects of polarization of the $Z$ boson are carried by its decay products, namely, the leptons through their angular distributions. We construct several kinematic variables using the negatively charged lepton as a reference and highlight its importance in observing the polarization of the parent gauge boson. We also propose new variables which utilize the observed asymmetries between the angular variables for the charged lepton coming from a parent longitudinal and transverse $Z$ boson. We do a full detector level simulation of the events and study the asymmetries that show the characteristic features of a longitudinal $Z$ boson and observe substantial differences between a Higgsino and gauginolike NLSP. This highlights the robustness of the constructed asymmetries. Our analysis is applicable to other BSM scenarios, which predict preferential production of longitudinally polarized gauge bosons as a consequence of the equivalence theorem.

\section{ACKNOWLEDGMENTS}

The work is partially supported by funding available from the Department of Atomic Energy, Government of India, for the Regional Centre for Accelerator-based Particle Physics (RECAPP), Harish-Chandra Research Institute (HRI). The research of J.D. is also partially supported by the INFOSYS scholarship for senior students at the Harish-Chandra Research Institute. We thank A. Ghosh, P.Konar, S. Mondal, and T. Samui for helpful comments, and K. Hagiwara for illuminating discussions.
[1] https://atlas.web.cern.ch/Atlas/GROUPS/PHYSICS/ CombinedSummaryPlots/SUSY.

[2] https://twiki.cern.ch/twiki/bin/view/CMSPublic/Physics ResultsSUS.

[3] N. Aghanim et al., Planck 2018 results. VI. Cosmological parameters (2018), https://inspirehep.net/literature/ 1682902.

[4] J. Fan and M. Reece, In Wino veritas? Indirect searches shed light on neutralino dark matter, J. High Energy Phys. 10 (2013) 124.

[5] M. Abdughani, J. Ren, and J. Zhao, Tev susy dark matter confronted with the current direct and indirect detection data, Eur. Phys. J. C 79, 146 (2019).

[6] G. Arcadi, M. Dutra, P. Ghosh, M. Lindner, Y. Mambrini, M. Pierre, S. Profumo, and F. S. Queiroz, The waning of the wimp? A review of models, searches, and constraints, Eur. Phys. J. C 78, 203 (2018).

[7] H. Baer, V. Barger, D. Sengupta, and X. Tata, Is natural higgsino-only dark matter excluded?, Eur. Phys. J. C 78, 838 (2018).

[8] M. Abdughani, L. Wu, and J. M. Yang, Status and prospects of light binohiggsino dark matter in natural SUSY, Eur. Phys. J. C 78, 4 (2018).

[9] S. P. Martin, A supersymmetry primer, Adv. Ser. Dir. High Energy Phys. 18, 1 (1998).
[10] M. Drees, R. Godbole, and P. Roy, Theory and Phenomenology of Sparticles: An Account of Four-Dimensional $N=1$ Supersymmetry in High Energy Physics (World Scientific. Singapore, 2004).

[11] G. F. Giudice and R. Rattazzi, Theories with gauge mediated supersymmetry breaking, Phys. Rep. 322, 419 (1999).

[12] J. Baur, N. Palanque-Delabrouille, C. Yèche, C. Magneville, and M. Viel, Lyman-alpha Forests cool warm dark matter, J. Cosmol. Astropart. Phys. 08 (2016) 012.

[13] A. Boyarsky, J. Lesgourgues, O. Ruchayskiy, and M. Viel, Lyman-alpha constraints on warm and on warm-plus-cold dark matter models, J. Cosmol. Astropart. Phys. 05 (2009) 012.

[14] L. Covi, Dark matter candidates: Axino and gravitino, in Proceedings, 46th Rencontres de Moriond on Electroweak Interactions and Unified Theories: La Thuile, Italy, 2011, pp. 381-388, https://indico.in2p3.fr/event/4403/session/12/ contribution/34/material/paper/0.pdf.

[15] P. Meade, M. Reece, and D. Shih, Prompt decays of general neutralino NLSPs at the tevatron, J. High Energy Phys. 05 (2010) 105.

[16] K. T. Matchev and S. D. Thomas, Higgs and $Z$ boson signatures of supersymmetry, Phys. Rev. D 62, 077702 (2000).

[17] A. M. Sirunyan et al., Combined search for electroweak production of charginos and neutralinos in proton-proton collisions at $\sqrt{s}=13 \mathrm{TeV}, \mathrm{J}$. High Energy Phys. 03 (2018) 160. 
[18] https://atlas.web.cern.ch/Atlas/GROUPS/PHYSICS/Combined SummaryPlots/SUSY/ATLAS_SUSY_EWSummary_GGM/ ATLAS_SUSY_EWSummary_GGM.png.

[19] H. Baer, V. Barger, P. Huang, D. Mickelson, A. Mustafayev, and $\mathrm{X}$. Tata, Radiative natural supersymmetry: Reconciling electroweak fine-tuning and the Higgs boson mass, Phys. Rev. D 87, 115028 (2013).

[20] H. Baer, V. Barger, P. Huang, D. Mickelson, A. Mustafayev, and $\mathrm{X}$. Tata, Naturalness, supersymmetry and light higgsinos: A snowmass whitepaper, in Proceedings, Community Summer Study 2013: Snowmass on the Mississippi (CSS2013): Minneapolis, MN, USA, 2013, https://inspirehep .net/record/1238302/files/arXiv:1306.2926.pdf.

[21] A. Mustafayev and X. Tata, Supersymmetry, naturalness, and light higgsinos, Indian J. Phys. 88, 991 (2014).

[22] H. Baer, V. Barger, P. Huang, A. Mustafayev, and X. Tata, Radiative Natural SUSY with a $125 \mathrm{GeV}$ Higgs Boson, Phys. Rev. Lett. 109, 161802 (2012).

[23] H. Baer, V. Barger, M. Savoy, and X. Tata, Multichannel assault on natural supersymmetry at the high luminosity LHC, Phys. Rev. D 94, 035025 (2016).

[24] H. Baer, V. Barger, J. S. Gainer, P. Huang, M. Savoy, D. Sengupta, and X. Tata, Gluino reach and mass extraction at the lhc in radiatively-driven natural susy, Eur. Phys. J. C 77, 499 (2017).

[25] H. Baer and X. Tata, Weak Scale Supersymmetry: From Superfields to Scattering Events (Cambridge University Press, Cambridge, England, 2006).

[26] M. Drees, M. M. Nojiri, D. P. Roy, and Y. Yamada, Light Higgsino dark matter, Phys. Rev. D 56, 276 (1997); Erratum, Phys. Rev. D 64, 039901 (2001).

[27] G. F. Giudice and A. Pomarol, Mass degeneracy of the Higgsinos, Phys. Lett. B 372, 253 (1996).

[28] A. Djouadi, The anatomy of electro-weak symmetry breaking. II. The Higgs bosons in the minimal supersymmetric model, Phys. Rep. 459, 1 (2008).

[29] L. Covi, J. Hasenkamp, S. Pokorski, and J. Roberts, Gravitino dark matter and general neutralino NLSP, J. High Energy Phys. 11 (2009) 003.

[30] J. Hasenkamp, General neutralino NLSP with gravitino dark matter vs. big bang nucleosynthesis, Ph.D. Thesis, Hamburg University, 2009.

[31] W. Porod, SPheno, A program for calculating supersymmetric spectra, SUSY particle decays and SUSY particle production at e+ e- colliders, Comput. Phys. Commun. 153, 275 (2003).

[32] W. Porod and F. Staub, SPheno 3.1: Extensions including flavour, $C P$-phases and models beyond the MSSM, Comput. Phys. Commun. 183, 2458 (2012).

[33] M. Aaboud et al., Search for pair production of higgsinos in final states with at least three $b$-tagged jets in $\sqrt{s}=13 \mathrm{TeV}$ $p p$ collisions using the ATLAS detector, Phys. Rev. D 98, 092002 (2018).

[34] A. M. Sirunyan et al., Search for Physics Beyond the Standard Model in Events with High-Momentum Higgs Bosons and Missing Transverse Momentum in Proton-Proton Collisions at 13 TeV, Phys. Rev. Lett. 120, 241801 (2018).

[35] M. Aaboud et al., Search for supersymmetry in events with four or more leptons in $\sqrt{s}=13 \mathrm{TeV} p p$ collisions with ATLAS, Phys. Rev. D 98, 032009 (2018).
[36] M. Aaboud et al., Search for squarks and gluinos in final states with jets and missing transverse momentum using $36 \mathrm{fb}^{-1}$ of $\sqrt{s}=13 \mathrm{TeV}$ pp collision data with the ATLAS detector, Phys. Rev. D 97, 112001 (2018).

[37] M. Aaboud et al. (ATLAS Collaboration), Search for chargino and neutralino production in final states with a Higgs boson and missing transverse momentum at $\sqrt{s}=13 \mathrm{TeV}$ with the ATLAS detector, Phys. Rev. D 100, 012006 (2019).

[38] A. M. Sirunyan et al., Search for new phenomena in final states with two opposite-charge, same-flavor leptons, jets, and missing transverse momentum in $\mathrm{pp}$ collisions at $\sqrt{s}=13 \mathrm{TeV}$, J. High Energy Phys. 03 (2018) 076.

[39] A. M. Sirunyan et al. (CMS Collaboration), Search for Physics beyond the Standard Model in Events with HighMomentum Higgs Bosons and Missing Transverse Momentum in Proton-Proton Collisions at 13 tev, Phys. Rev. Lett. 120, 241801 (2018).

[40] A. M. Sirunyan et al., Combined measurements of Higgs boson couplings in proton-proton collisions at $\sqrt{s}=13 \mathrm{TeV}$, Eur. Phys. J. C 79, 421 (2019).

[41] S. Chatrchyan et al., Observation of a new boson at a mass of $125 \mathrm{GeV}$ with the CMS experiment at the LHC, Phys. Lett. B 716, 30 (2012).

[42] A. Arbey, M. Battaglia, A. Djouadi, F. Mahmoudi, and J. Quevillon, Implications of a 125 gev higgs for supersymmetric models, Phys. Lett. B 708, 162 (2012).

[43] LEPSUSYWG et al., LEP2 SUSY Working Group, http:// lepsusy.web.cern.ch/lepsusy/.

[44] M. Drees, H. Dreiner, D. Schmeier, J. Tattersall, and J. S. Kim, CheckMATE: Confronting your favourite new physics model with LHC data, Comput. Phys. Commun. 187, 227 (2015).

[45] J. Alwall, R. Frederix, S. Frixione, V. Hirschi, F. Maltoni, O. Mattelaer, H. S. Shao, T. Stelzer, P. Torrielli, and M. Zaro, The automated computation of tree-level and next-to-leading order differential cross sections, and their matching to parton shower simulations, J. High Energy Phys. 07 (2014) 079.

[46] N. D. Christensen, P. de Aquino, N. Deutschmann, C. Duhr, B. Fuks, C. Garcia-Cely, O. Mattelaer, K. Mawatari, B. Oexl, and Y. Takaesu, Simulating spin-3/2 particles at colliders, Eur. Phys. J. C 73, 2580 (2013).

[47] T. Sjostrand, S. Mrenna, and P.Z. Skands, PYTHIA6.4 physics and manual, J. High Energy Phys. 05 (2006) 026.

[48] PYTHIA, http://home.thep.lu.se/ torbjorn/pythia81html/ Welcome.html.

[49] J. de Favereau, C. Delaere, P. Demin, A. Giammanco, V. Lemaître, A. Mertens, and M. Selvaggi, DELPHES3, A modular framework for fast simulation of a generic collider experiment, J. High Energy Phys. 02 (2014) 057.

[50] M. Cacciari, G. P. Salam, and Gr. Soyez, The Anti- $k_{t}$ jet clustering algorithm, J. High Energy Phys. 04 (2008) 063.

[51] M. Cacciari, G. P. Salam, and G. Soyez, FastJet user manual, Eur. Phys. J. C 72, 1896 (2012).

[52] H.-C. Cheng and Z. Han, Minimal kinematic constraints and MT2, J. High Energy Phys. 12 (2008) 063.

[53] W. Beenakker, R. Hopker, and M. Spira, PROSPINO: A program for the production of supersymmetric particles in next-to-leading order QCD (1996), http://www.thphys.uniheidelberg.de/ plehn/index.php?show=prospino\&visible= tools. 
[54] W. Beenakker, C. Borschensky, M. Krämer, A. Kulesza, E. Laenen, S. Marzani, and J. Rojo, NLO+NLL squark and gluino production cross-sections with threshold-improved parton distributions, Eur. Phys. J. C 76, 53 (2016).

[55] https://twiki.cern.ch/twiki/bin/view/LHCPhysics/LHCHXS WG1HELHCXsecs.

[56] M. Grazzini, S. Kallweit, D. Rathlev, and M. Wiesemann, $W^{ \pm} Z$ production at hadron colliders in NNLO QCD, Phys. Lett. B 761, 179 (2016).

[57] F. Cascioli, T. Gehrmann, M. Grazzini, S. Kallweit, P. Maierhöfer, A. von Manteuffel, S. Pozzorini, D. Rathlev, L. Tancredi, and E. Weihs, ZZ production at hadron colliders in NNLO QCD, Phys. Lett. B 735, 311 (2014).

[58] M. Czakon, P. Fiedler, and A. Mitov, Total Top-Quark Pair-Production Cross Section at Hadron Colliders Through $O\left(\frac{4}{S}\right)$, Phys. Rev. Lett. 110, 252004 (2013).

[59] LHC TTbar NNLO, https://twiki.cern.ch/twiki/bin/view/ LHCPhysics/TtbarNNLO\#Top_quark_pair_cross_sections_at.

[60] M. J. Duncan, G. L. Kane, and W. W. Repko, Ww physics at future colliders, Nucl. Phys. B272, 517 (1986).
[61] M. Aaboud et al., Measurement of $W^{ \pm} Z$ production cross sections and gauge boson polarisation in $p p$ collisions at $\sqrt{s}=13 \mathrm{TeV}$ with the ATLAS detector, Eur. Phys. J. C 79, 535 (2019).

[62] P. Meade, M. Reece, and D. Shih, Long-lived neutralino NLSPs, J. High Energy Phys. 10 (2010) 067.

[63] G. Brooijmans et al., Les Houches 2017: Physics at $\mathrm{TeV}$ colliders new physics working group report, in 10th Les Houches Workshop on Physics at TeV Colliders (PhysTeV 2017) Les Houches, France, 2017 (2018), http://lss.fnal.gov/archive/2017/conf/fermilab-conf-17-664ppd.pdf.

[64] J. R. Andersen et al., Les Houches 2017: Physics at $\mathrm{TeV}$ colliders Standard Model working group report, in 10th Les Houches Workshop on Physics at TeV Colliders (PhysTeV 2017) Les Houches, France, 2017 (2018), http:// lss.fnal.gov/archive/2018/conf/fermilab-conf-18-122-cd-t .pdf.

[65] F. James, Statistical Methods in Experimental Physics (World Scientific, Singapore, 2006). 